\title{
Unique recovery of lower order coefficients for hyperbolic equations from data on disjoint sets
}

\section{Kian, Yavar}

2019-08-05

Kian , Y , Kurylev , Y, Lassas , M \& Oksanen , L 2019 , ' Unique recovery of lower order coefficients for hyperbolic equations from data on disjoint sets ' , Journal of Differential Equations , vol. 267 , no. 4 , pp. 2210-2238 . https://doi.org/10.1016/j.jde.2019.03.008

http://hdl.handle.net/10138/332834

https://doi.org/10.1016/j.jde.2019.03.008

cc_by_nc_nd

acceptedVersion

Downloaded from Helda, University of Helsinki institutional repository.

This is an electronic reprint of the original article.

This reprint may differ from the original in pagination and typographic detail.

Please cite the original version. 


\title{
UNIQUE RECOVERY OF LOWER ORDER COEFFICIENTS FOR HYPERBOLIC EQUATIONS FROM DATA ON DISJOINT SETS
}

\author{
YAVAR KIAN, YAROSLAV KURYLEV, MATTI LASSAS, \\ AND LAURI OKSANEN
}

\begin{abstract}
We consider a restricted Dirichlet-to-Neumann map $\Lambda_{\mathcal{S}, \mathcal{R}}^{T}$ associated with the operator $\partial_{t}^{2}-\Delta_{g}+A+q$ where $\Delta_{g}$ is the Laplace-Beltrami operator of a Riemannian manifold $(M, g)$, and $A$ and $q$ are a vector field and a function on $M$. The restriction $\Lambda_{\mathcal{S}, \mathcal{R}}^{T}$ corresponds to the case where the Dirichlet traces are supported on $(0, T) \times \mathcal{S}$ and the Neumann traces are restricted on $(0, T) \times \mathcal{R}$. Here $\mathcal{S}$ and $\mathcal{R}$ are open sets, which may be disjoint, on the boundary of $M$. We show that $\Lambda_{\mathcal{S}, \mathcal{R}}^{T}$ determines uniquely, up the natural gauge invariance, the lower order terms $A$ and $q$ in a neighborhood of the set $\mathcal{R}$ assuming that $\mathcal{R}$ is strictly convex and that the wave equation is exactly controllable from $\mathcal{S}$ in time $T / 2$. We give also a global result under a convex foliation condition. The main novelty is the recovery of $A$ and $q$ when the sets $\mathcal{R}$ and $\mathcal{S}$ are disjoint. We allow $A$ and $q$ to be non-self-adjoint, and in particular, the corresponding physical system may have dissipation of energy.
\end{abstract}

\section{INTRODUCTION}

Let $(M, g)$ be a smooth, connected and compact Riemannian manifold of dimension $n$ with nonempty boundary $\partial M$, let $A$ be a smooth complex valued vector field on $M$, and let $q$ be a smooth complex valued function on $M$. We consider the wave equation with Dirichlet data $f \in C_{0}^{\infty}((0, \infty) \times \partial M)$,

$$
\begin{cases}\left(\partial_{t}^{2}-\Delta_{g}+A(x)+q(x)\right) u(t, x)=0, & \text { in }(0, \infty) \times M \\ \left.u\right|_{(0, \infty) \times \partial M}=f, & \text { in }(0, \infty) \times \partial M \\ \left.u\right|_{t=0}=\left.\partial_{t} u\right|_{t=0}=0, & \text { in } M,\end{cases}
$$

Date: December 1, 2018.

1991 Mathematics Subject Classification. Primary: 35R30.

Key words and phrases. Inverse problems, wave equation, partial data. 
and denote by $u_{f}=u(t, x)$ the solution of (1). For open and nonempty sets $\mathcal{S}, \mathcal{R} \subset \partial M$ and $T \in(0, \infty]$ we define the response operator,

$$
\Lambda_{\mathcal{S}, \mathcal{R}}^{T}:\left.f \mapsto\left(\partial_{\nu} u_{f}-\frac{1}{2}(A, \nu)_{g} u_{f}\right)\right|_{(0, T) \times \mathcal{R}}, \quad f \in C_{0}^{\infty}((0, T) \times \mathcal{S}) .
$$

Here $\nu$ is the exterior unit normal vector field on $\partial M$, and $(A, \nu)_{g}$ is the inner product of $A$ and $\nu$. We use real inner products throughout the paper. If $A(x)=\sum_{j=1}^{n} A^{j}(x) \partial_{j}$ in local coordinates, then $(A, \nu)_{g}$ is given by $g_{j k} A^{j} \nu^{k}$ locally.

When $f$ is regarded as a boundary source, the operator $\Lambda_{\mathcal{S}, \mathcal{R}}^{T}$ models boundary measurements for the wave equation with sources on the set $(0, T) \times \mathcal{S}$ and the waves being observed on $(0, T) \times \mathcal{R}$. We consider the inverse boundary value problem to determine the manifold $(M, g)$, the vector field $A$ and the potential $q$ from $\Lambda_{\mathcal{S}, \mathcal{R}}^{T}$.

We have studied previously the determination of the geometry $(M, g)$ in the case that $A=0$ and $q=0$, see [30]. This corresponds to the recovery of the leading order terms in the wave equation up to isometries. It appears to us that when the sets $\mathcal{S}$ and $\mathcal{R}$ are disjoint, the recovery of the lower order terms $A$ and $q$ is more complicated than the recovery of the leading order terms, and this is the main focus of the present paper. It should be emphasized that if $\mathcal{S}$ and $\mathcal{R}$ are disjoint and if $\Lambda_{\mathcal{S}, \mathcal{R}}^{\infty}$ is known only at a fixed frequency in the sense that its Fourier transform in time is given at fixed point, then it is not possible to recover even the geometry $(M, g)$, see [12].

In order to recover $A$ and $q$, we develop a new technique that is based on exploiting strict convexity of the set $\mathcal{R}$. We recall that $\mathcal{R}$ is said to be strictly convex if its second fundamental form is positive definite, that is, $I I(v, v)>0$ for all $v \in T_{x} \mathcal{R}$ and $x \in \mathcal{R}$, where

$$
I I(v, v)=\left(\nabla_{v} \nu, v\right)_{g}
$$

and $\nabla$ is the covariant derivative on $(M, g)$. Using the convexity we construct a boundary source $f$ such that, at time $t=T$, the corresponding solution $u_{f}$ is essentially localized in a small set near $\mathcal{R}$.

The lower order terms $A$ and $q$ can be determined only up to the action of a group gauge transformations, that we will describe next. Let $\kappa$ be a smooth nowhere vanishing complex valued function on $M$ satisfying $\kappa=1$ on $\mathcal{R}$. The response operator $\Lambda_{\mathcal{S}, \mathcal{R}}^{T}$ does not change under the transformation $(A, q) \mapsto\left(A_{\kappa}, q_{\kappa}\right)$ where

$$
A_{\kappa}=A+2 \kappa^{-1} \operatorname{grad}_{g} \kappa, \quad q_{\kappa}=q+\kappa\left(A-\Delta_{g}\right) \kappa^{-1}
$$

and $\operatorname{grad}_{g}$ is the gradient on $(M, g)$. We refer to [25] for a similar computation in the self-adjoint case. When $\mathcal{U} \subset M$ is a neighborhood 
of $\mathcal{R}$, we write

$$
\mathcal{G}_{\mathcal{U}, \mathcal{R}}(A, q)=\left\{\left(\left.A_{\kappa}\right|_{\mathcal{U}},\left.q_{\kappa}\right|_{\mathcal{U}}\right) ; \kappa \in C^{\infty}(\overline{\mathcal{U}}),|\kappa|>0,\left.\kappa\right|_{\mathcal{R}}=1\right\}
$$

for the orbit of the group of gauge transformations on $\mathcal{U}$.

We recall that the wave equation $(1)$ is said to be exactly controllable from $\mathcal{S}$ in time $T$ if the map

$$
f \mapsto\left(u_{f}(T), \partial_{t} u_{f}(T)\right): L^{2}((0, T) \times \mathcal{S}) \rightarrow L^{2}(M) \times H^{-1}(M),
$$

is surjective. If there is such $T>0$, then we say that (1) is exactly controllable from $\mathcal{S}$. The exact controllability can be characterized in terms of the billiard flow of the manifold $(M, g)$ [3, 9]. The geometric characterization says roughly that all unit speed geodesics, continued by reflection on $\partial M \backslash \mathcal{S}$, must exit $M$ through $\mathcal{S}$ during time $T$. In particular, the geometric characterization implies that the exact controllability does not depend on the lower order terms $A$ and $q$.

In this paper we show the following theorem:

Theorem 1. Let $\mathcal{S} \subset \partial M$ be open and suppose that the wave equation (1) is exactly controllable from $\mathcal{S}$ in time $T>0$. Let $\mathcal{R} \subset \partial M$ be open and strictly convex. For $j=1,2$, we fix $A_{j} \in C^{\infty}(M ; T M)$ $q_{j} \in C^{\infty}(M ; \mathbb{C})$, we denote by $\Lambda_{j, \mathcal{S}, \mathcal{R}}^{2 T}$ the response operator at time $2 T$ associated with (1) with $A=A_{j}$ and $q=q_{j}$. Then assuming that

$$
\Lambda_{1, \mathcal{S}, \mathcal{R}}^{2 T}=\Lambda_{2, \mathcal{S}, \mathcal{R}}^{2 T}
$$

there is a neighborhood $\mathcal{U} \subset M$ of $\mathcal{R}$, independent of $\left(A_{1}, q_{1}\right)$ and $\left(A_{2}, q_{2}\right)$, such that

$$
\left(\left.A_{1}\right|_{\mathcal{U}},\left.q_{1}\right|_{\mathcal{U}}\right) \in \mathcal{G}_{\mathcal{U}, \mathcal{R}}\left(A_{2}, q_{2}\right) .
$$

We show also a global uniqueness result under the assumption that there is a convex foliation similar to that in [35]. We assume that $\Sigma_{s}$, $s \in(0,1]$, satisfy the following:

(F1) $\Sigma_{s} \subset M^{\text {int }}$ is a smooth manifold of codimension one.

(F2) The union $\Omega_{s}=\bigcup_{r \in(0, s)} \Sigma_{r} \subset M^{\text {int }}$ is open and connected, and $\Omega_{r} \subset \Omega_{s}$ when $r<s$.

(F3) $\partial \Omega_{s}=\Sigma_{s} \cup \mathcal{R}_{s}$ and $\mathcal{R}_{s} \subset \mathcal{R}$ where $\mathcal{R}_{s}=\overline{\Omega_{s}} \cap \partial M$.

(F4) $\Sigma_{s}$ is strictly convex as a subset of $\partial M_{s}$ where

$$
M_{s}=M \backslash\left(\Omega_{s} \cup \mathcal{R}_{s}\right) .
$$

(F5) The Hausdorff distances satisfy $\operatorname{dist}\left(\Omega_{r}, \Omega_{s}\right) \rightarrow 0$ as $r \rightarrow s$.

(F6) There is a set $\mathcal{R}_{0} \subset \mathcal{R}$ such that $\operatorname{dist}\left(\Omega_{s}, \mathcal{R}_{0}\right) \rightarrow 0$ as $s \rightarrow 0$.

Furthermore, to simplify the notation, we assume

(F7) $\mathcal{R}=\bigcup_{s \in(0,1]} \mathcal{R}_{s}$. 
Theorem 2. Let $\mathcal{S} \subset \partial M$ be open and suppose that the wave equation (1) is exactly controllable from $\mathcal{S}$. Let $\mathcal{R} \subset \partial M$ be open and strictly convex and let $\Sigma_{s}, s \in(0,1]$, be a convex foliation satisfying (F1)-(Fr). Then (4) implies that there exists $\mathcal{U} \subset M$ an open set of $M$ containing $\bar{\Omega}_{1}$ such that

$$
\left(\left.A_{1}\right|_{\mathcal{U}},\left.q_{1}\right|_{\mathcal{U}}\right) \in \mathcal{G}_{\mathcal{U}, \mathcal{R}}\left(A_{2}, q_{2}\right) .
$$

In Section 5 we show that, in the above theorem, exact controllability from $\mathcal{S}$ can be replaced with exact controllability from $\mathcal{R}$. Our result is new even in the following case:

Example 1. Let $(M, g)$ be the Euclidean unit disk $\{z \in \mathbb{C} ;|z| \leq 1\}$. Let $\epsilon>0$ and define $\mathcal{R}=\left\{e^{i \theta} ; \theta \in(-\epsilon, \pi+\epsilon)\right\}$. Let $\mathcal{S} \subset \partial M$ be open and nonempty. Then $\Lambda_{\mathcal{S}, \mathcal{R}}^{\infty}$ determines $A$ and $q$, up to the gauge transformations, in the convex hull of $\mathcal{R}$.

Example 2. Let $(M, g)$ be the Euclidean annulus

$$
\left\{z \in \mathbb{C} ; r_{1} \leq|z| \leq r_{2}\right\}, \quad \text { with } 0<r_{1}<r_{2} \text {. }
$$

Let $\epsilon>0$ and define $\mathcal{R}=\left\{r_{2} e^{i \theta} ; \theta \in(-\epsilon, \pi+\epsilon)\right\}$. Let $\mathcal{S} \subset \partial M$ be open and nonempty and assume that $\mathcal{S}$ satisfies the geometrical control condition of [3], for example, $\mathcal{S}=\left\{z \in \mathbb{C} ;|z|=r_{1}\right\}$. Then $\Lambda_{\mathcal{S}, \mathcal{R}}^{\infty}$ determines $A$ and $q$, up to the gauge transformations, in the convex hull of $\mathcal{R}$.

Let us also point out that we could use a time continuation argument analogous to [30, Lemma 4] and prove Theorem 2 also for measurements on a long enough but finite time interval.

Our proof is based on the Boundary Control (BC) method. The BC method was introduced by Belishev [4], and it was first used in a geometric context in [5]. Stability properties of the method are discussed in [2] and in the recent preprint [7]. First order perturbations have been considered in the self-adjoint case in [20, 27], and in the non-selfadjoint case in $[26,28]$. All the above results assume that $\mathcal{S}=\mathcal{R}$. The case of disjoint $\mathcal{S}$ and $\mathcal{R}$ was first considered in the above mentioned [30] where no first order perturbation was present.

The previous version of the BC method [30, with data on disjoint sets, was based on recovering distance functions and did not use localization of waves to delta-type functions, a technique that we develop in the present paper. Needless to say, distance functions contain no information on lower order terms. Moreover, the proofs in above mentioned [20, 26, 27, 28] make a heavy use of the assumption $\mathcal{S}=\mathcal{R}$, and therefore they do not generalize to the present setting with disjoint $\mathcal{S}$ and $\mathcal{R}$. 
In addition to [30], we are aware of only two results on inverse boundary value problems with disjoint data analogous to the case $\overline{\mathcal{S}} \cap \overline{\mathcal{R}}=\emptyset$. Rakesh [33] considers a wave equation on a one-dimensional interval with sources supported on one end of the interval and the waves observed on the other end, and Imanuvilov, Uhlmann, and Yamamoto [19] proved that a zeroth order term in a Schrödinger equation on a two-dimensional domain homeomorphic to a disk, whose boundary is partitioned into eight parts $\Gamma_{1}, \Gamma_{2}, \ldots, \Gamma_{8}$ in the clockwise order, is determined by boundary measurements with Dirichlet data supported on $\mathcal{S}=\Gamma_{2} \cup \Gamma_{6}$ and the Neumann trace observed on $\mathcal{R}=\Gamma_{4} \cup \Gamma_{8}$.

Let us mention also the result on recovery of a conformal scaling factor in the metric tensor given the Dirichlet-to-Neumann map [37] that, analogously to our result, uses local convexity of the boundary. The proof [37] is based on a reduction to the boundary rigidity result [36] and this approach seems to require that $\mathcal{S}=\mathcal{R}$. Let us also mention that, using the equivalence between inverse problems for hyperbolic equation and inverse problems stated for other equations described in [22, 24, the result of this paper can also be applied in other context for different types of equations.

A vast majority of results on inverse boundary value problems assume that $\overline{\mathcal{S}} \cap \overline{\mathcal{R}} \neq \emptyset$. For this type of non-disjoint, partial data results, we refer to [10, 11, 13, 15, 16, 17, 18, 23].

1.1. Outline of the paper. In Section 2.1 we first prove a version of Blagoveščenskiı̌'s identity that, given the response operator $\Lambda_{\mathcal{S}, \mathcal{R}}^{2 T}$, allows us to compute inner products of the form

$$
\left\langle v_{\phi}(T, \cdot), u_{f}(T, \cdot)\right\rangle_{L^{2}(M)}
$$

where $u_{f}$ is the solution of $(1)$, with $f$ supported in $(0,2 T) \times \mathcal{S}$, and $v_{\phi}$ is the solution of a dual problem, see $10 p$ below, with the source $\phi$ supported in $(0,2 T) \times \mathcal{R}$.

It follows from approximate controllability, as recalled in Section 2.2 , that if for a fixed $f$ the inner products (7) vanish for all $\phi$ in $C_{0}^{\infty}((0,2 T) \times \mathcal{R})$, then $u_{f}(T, \cdot)$ must be supported outside the set of points $x \in M$ satisfying $d(x, \mathcal{R})<T$.

A key idea in the proof is to use a more refined argument to show that for a point $x \in M$ near $\mathcal{R}$ we can choose a set $\mathcal{B} \subset(0,2 T) \times \mathcal{R}$ such that if (7) vanish for all $\phi$ in $C_{0}^{\infty}(\mathcal{B})$, then $u_{f}(T, \cdot)$ must be supported outside a set that isolates a neighbourhood of $x$ as in Figure 2 in Section 3.1. This construction uses the convexity of $\mathcal{R}$ and it is carried out in Section 3.1. 
Using the assumption that the wave equation (1) is exactly controllable from $\mathcal{S}$ in time $T>0$, we then show in Section 3.2 that it is possible to construct a sequence of sources $\left(f_{k}\right)_{k \in \mathbb{N}}$ in $L^{2}((0, T) \times \mathcal{S})$ so that, when restricted near $\mathcal{R}$, the functions $u_{f_{k}}(T, \cdot)$ converge to a point mass $\kappa \delta_{x}$ at $x$, with $\kappa$ a nonzero constant.

We finish the proof of Theorem 1 in Section 3.3. The idea is to substitute $u_{f_{k}}(T, \cdot)$ in (7) and recover $\kappa v_{\phi}(T, x)$ after passing to the limit. Here the constant $\kappa$ depends on the choice of the point $x$, and some technical work is required in order to recover $\kappa(x) v_{\phi}(T, x)$, with $\kappa$ a smooth function near $\mathcal{R}$. Having the functions $\kappa(x) v_{\phi}(T, x)$ at hand, it is straightforward to find out which wave equation they satisfy. This will give us $A_{\kappa}$ and $q_{\kappa}$ as in (2).

The global result in Theorem 2 is proven in Section 4 by iterating the local construction along the convex foliation. Finally in Section 5, we give some complementary results.

\section{TOOLS FOR THE INVERSE PROBLEM}

In this section we present the two main components of the Boundary Control method: an integration by parts technique originating from Blagoveščenskiı̀'s study of the $1+1$ dimensional wave equation [6], and a density result based on the hyperbolic unique continuation result by Tataru 38].

2.1. Blagoveščenskiı̌'s identity. Let $\Gamma \subset \partial M$ and $B \subset M^{\text {int }}$ be open, and let $\kappa: B \rightarrow \mathbb{C}$ be a smooth function satisfying

$$
\kappa(x) \neq 0, \quad x \in B
$$

We define for $f \in \mathcal{C}_{0}^{\infty}((0, \infty) \times S)$,

$$
\Lambda_{\Gamma} f=\left.\left(\partial_{\nu} u-\frac{1}{2}(A, \nu)_{g} u\right)\right|_{(0, \infty) \times \Gamma}, \quad \mathcal{T}_{B, \kappa} f=\left.\kappa u\right|_{(0, \infty) \times B},
$$

where $u$ is the solution of $(1)$, and write $\mathcal{T}_{B}=\mathcal{T}_{B, \kappa}$ when considering a fixed $\kappa$.

For all $\tau>0$ we define $K_{\Gamma}^{\tau}$ and $K_{B, \kappa}^{\tau}$, for any $f \in C_{0}^{\infty}((0, \infty) \times \mathcal{S})$, $(t, x) \in(0,+\infty) \times \partial M$ and $(t, y) \in(0,+\infty) \times B$, by

$$
\begin{aligned}
K_{\Gamma}^{\tau} f(t, x) & =\Lambda_{\Gamma} J^{\tau} f(\tau-t, x)-\widetilde{J}^{\tau} \Lambda_{\Gamma} f(t, x), \\
K_{B, \kappa}^{\tau} f(t, y) & =\mathcal{T}_{B, \kappa} J^{\tau} f(\tau-t, y)-\widetilde{J}^{\tau} \mathcal{T}_{B, \kappa} f(t, y),
\end{aligned}
$$

where $J^{\tau} \psi(t, x)=\frac{1}{2} \int_{\tau-t}^{t+\tau} \psi(s, x) d s$ and $\widetilde{J}^{\tau} \psi(t, x)=\frac{1}{2} \int_{t}^{2 \tau-t} \psi(s, x) d s$. 
Let us now consider the adjoint problems

$$
\begin{gathered}
(10) \begin{cases}\partial_{t}^{2} v-\Delta_{g} v-\bar{A} v+\left(\bar{q}-\operatorname{div}_{g} \bar{A}\right) v=0, & \text { in }(0, \infty) \times M, \\
\left.v\right|_{(0, \infty) \times \partial M}=\phi, & \text { in }(0, \infty) \times \partial M, \\
\left.v\right|_{t=0}=\left.\partial_{t} v\right|_{t=0}=0, & \text { in } M,\end{cases} \\
\left(1 1 \left\{\begin{array}{ll}
\partial_{t}^{2} w-\Delta_{g} w-\bar{A} w+\left(\bar{q}-\operatorname{div}_{g} \bar{A}\right) w=H, & \text { in }(0, \infty) \times M, \\
\left.w\right|_{(0, \infty) \times \partial M}=0, & \text { in }(0, \infty) \times \partial M, \\
\left.w\right|_{t=0}=\left.\partial_{t} w\right|_{t=0}=0, & \text { in } M,
\end{array}\right.\right.
\end{gathered}
$$

where $H \in C_{0}^{\infty}((0,+\infty) \times B), \phi \in C_{0}^{\infty}((0, \infty) \times \partial M)$, div $\operatorname{din}_{g}$ the divergence on $(M, g)$. We fix $v_{f}$ (resp. $\left.u_{f}, w_{H}\right)$ the unique solution of (10) (resp. (1), (11)) lying in $C^{1}\left((0,+\infty) ; L^{2}(M)\right) \cap C\left((0,+\infty) ; H^{1}(M)\right)$. Now let us consider the following identity

Lemma 1 (Blagoveščenskiu type identity). Let $\tau>0$ and let $\Gamma \subset \partial M$ and $B \subset M^{\text {int }}$ be open. Then for functions $f \in L_{\text {loc }}^{2}((0,+\infty) \times \mathcal{S})$, $\phi \in L_{l o c}^{2}((0, \infty) \times \Gamma), H \in L_{l o c}^{2}((0,+\infty) \times B)$ we have

$$
\begin{aligned}
\left\langle v_{\phi}(\tau, \cdot), u_{f}(\tau, \cdot)\right\rangle_{L^{2}(M)} & =\left\langle\phi, K_{\Gamma}^{\tau} f\right\rangle_{L^{2}((0, \tau) \times \Gamma)} . \\
\left\langle w_{\bar{\kappa} H}(\tau, \cdot), u_{f}(\tau, \cdot)\right\rangle_{L^{2}(M)} & =\left\langle H, K_{B, \kappa}^{\tau} f\right\rangle_{L^{2}((0, \tau) \times \Gamma)} .
\end{aligned}
$$

Proof. Since the proof of (12) and (13) are similar, we will only treat (12). Without loss of generality and by density, we assume that $f \in$ $C_{0}^{\infty}((0,+\infty) \times \mathcal{S}), \phi \in C_{0}^{\infty}((0, \infty) \times \Gamma)$. For $t \in(0, \tau)$ and $s \in(0,2 \tau)$, we start by considering

$$
S(t, s)=\left\langle v_{\phi}(t, \cdot), u_{f}(s, \cdot)\right\rangle_{L^{2}(M)} \cdot
$$

Recall that

$$
\begin{aligned}
\left(\partial_{t}^{2}-\right. & \left.\partial_{s}^{2}\right) S(t, s) \\
= & \left\langle\left(\Delta_{g}+\bar{A}-\left(\bar{q}-\operatorname{div}_{g} \bar{A}\right)\right) v_{\phi}(t, \cdot), u_{f}(s, \cdot)\right\rangle_{L^{2}(M)} \\
& -\left\langle v_{\phi}(t, \cdot),\left(\Delta_{g}-A-q\right) u_{f}(s, \cdot)\right\rangle_{L^{2}(M)} \\
= & \left\langle\partial_{\nu} v_{\phi}(t, \cdot)+\frac{1}{2}(\bar{A}, \nu)_{g} v_{\phi}(t, \cdot), f(s, \cdot)\right\rangle_{L^{2}(\partial M)} \\
& -\left\langle\phi(t, \cdot), \Lambda_{\Gamma} f(s, \cdot)\right\rangle_{L^{2}(\partial M)} .
\end{aligned}
$$

Thus, fixing

$$
\begin{aligned}
F(t, s)= & \left\langle\partial_{\nu} v_{\phi}(t, \cdot)+\frac{1}{2}(\bar{A}, \nu)_{g} v_{\phi}(t, \cdot), f(s, \cdot)\right\rangle_{L^{2}(\partial M)} \\
& -\left\langle\phi(t, \cdot), \Lambda_{\Gamma} f(s, \cdot)\right\rangle_{L^{2}(\partial M)}
\end{aligned}
$$


we deduce that the function $S$ satisfies the $1+1$ dimensional wave equation

$$
\begin{cases}\partial_{t}^{2} S-\partial_{s}^{2} S=F, & \text { in }(0, \tau) \times(0,2 \tau) \\ S(0, \cdot)=0, \quad \partial_{t} S(0, \cdot)=0, & \text { in }(0, \tau) \\ S(\cdot, 0)=0, \quad \partial_{s} S(\cdot, 0)=0, & \text { in }(0,2 \tau)\end{cases}
$$

We solve this wave equation on the triangle with corners $(\tau, \tau),(0,0)$ and $(0,2 \tau)$, and obtain

$$
\begin{aligned}
& \left\langle v_{\phi}(\tau, \cdot), u_{f}(\tau, \cdot)\right\rangle_{L^{2}(M)} \\
& =\frac{1}{2} \int_{0}^{\tau} \int_{t}^{2 \tau-t}\left\langle\partial_{\nu} v_{\phi}(t, \cdot)+\frac{1}{2}(\bar{A}, \nu)_{g} v_{\phi}(t, \cdot), f(s, \cdot)\right\rangle_{L^{2}(\partial M)} d s d t \\
& -\frac{1}{2} \int_{0}^{\tau} \int_{t}^{2 \tau-t}\left\langle\phi(t, \cdot), \Lambda_{\Gamma} f(s, \cdot)\right\rangle_{L^{2}(\partial M)} d s d t .
\end{aligned}
$$

Now, for $h \in C_{0}^{\infty}((-\infty, \tau) \times \mathcal{S})$, let $u=R_{\tau} u_{R_{\tau} h}$ and $v=v_{\phi}$ where $R_{\tau}$ is the time reversal operator defined by $R_{\tau} h(t, x)=h(\tau-t, x)$. Then

$$
\begin{aligned}
& \left\langle h, \partial_{\nu} v+\frac{1}{2}(\bar{A}, \nu)_{g} v\right\rangle_{L^{2}((0, \tau) \times \partial M)}-\left\langle\partial_{\nu} u-\frac{1}{2}(A, \nu)_{g} u, \phi\right\rangle_{L^{2}((0, \tau) \times \partial M)} \\
& =\left\langle\left(\partial_{t}^{2}-\Delta_{g}+A+q\right) u, v\right\rangle_{L^{2}((0, \tau) \times M)} \\
& \quad-\left\langle u,\left(\partial_{t}^{2}-\Delta_{g}-\bar{A}+\left(\bar{q}-\operatorname{div}_{g} \bar{A}\right)\right) v\right\rangle_{L^{2}((0, \tau) \times M)}=0 .
\end{aligned}
$$

Therefore, we have

$$
\left\langle h, \partial_{\nu} v_{\phi}+\frac{1}{2}(\bar{A}, \nu)_{g} v_{\phi}\right\rangle_{L^{2}((0, \tau) \times \partial M)}=\left\langle R_{\tau} \Lambda_{\Gamma} R_{\tau} h, \phi\right\rangle_{L^{2}((0, \tau) \times \partial M)} .
$$

Fixing

$$
h(t, \cdot):=\int_{t}^{2 \tau-t} f(s, \cdot) d s
$$

we find

$$
\begin{aligned}
& \frac{1}{2} \int_{0}^{\tau} \int_{t}^{2 \tau-t}\left\langle\partial_{\nu} v_{\phi}(t, \cdot)+\frac{1}{2}(\bar{A}, \nu)_{g} v_{\phi}(t, \cdot), f(s, \cdot)\right\rangle_{L^{2}(\partial M)} d s d t \\
& =\frac{1}{2} \int_{0}^{\tau}\left\langle\partial_{\nu} v_{\phi}(t, \cdot)+\frac{1}{2}(\bar{A}, \nu)_{g} v_{\phi}(t, \cdot), h(t, \cdot)\right\rangle_{L^{2}(\partial M)} d t \\
& =\frac{1}{2} \int_{0}^{\tau}\left\langle\phi(t, \cdot), R_{\tau} \Lambda_{\Gamma} R_{\tau} h(t, \cdot)\right\rangle_{L^{2}(\partial M)} d t \\
& =\int_{0}^{\tau}\left\langle\phi(t, \cdot), R_{\tau} \Lambda_{\Gamma} J^{\tau} f(t, \cdot)\right\rangle_{L^{2}(\partial M)} d t .
\end{aligned}
$$

Combining this with 14 , we deduce 12 . 
2.2. Approximate controllability. Next we consider approximate controllability on a domain of influence. Let $T>0, \Gamma \subset \partial M$ and $B \subset M^{\text {int }}$ be open, and let $\mathcal{V}=\Gamma$ or $\mathcal{V}=B$. Let $h: \overline{\mathcal{V}} \rightarrow \mathbb{R}$ be piecewise continuous, and define the domain of influence

$$
M(\mathcal{V}, h)=\left\{x \in M ; \inf _{y \in \mathcal{V}}(d(x, y)-h(y)) \leq 0\right\},
$$

where $d$ is the distance function on $(M, g)$. Moreover, we write

$$
\mathcal{B}(\mathcal{V}, h ; T)=\{(t, y) \in(0, \infty) \times \mathcal{V} ; T-h(y)<t\} .
$$

We extend the notations $M(\mathcal{V}, h)$ and $\mathcal{B}(\mathcal{V}, h ; T)$ for constants $h \in \mathbb{R}$ by interpreting $h$ as a constant function. Moreover, we define $M(x, h)$ by $M(\{x\}, h)$ for points $x \in \partial M$.

We have the following approximate controllability result that is analogous to [30, Lemma 5] and [27, Lemma 2.5].

Lemma 2. Let $T>0, \Gamma \subset \partial M$ and $h: \Gamma \rightarrow \mathbb{R}$ to be piecewise continuous. Then, the set

$$
\left\{v_{\phi}(T, \cdot) ; \phi \in \mathcal{C}_{0}^{\infty}(\mathcal{B}(\Gamma, h ; T))\right\}
$$

is dense in $L^{2}(M(\Gamma, h))=\left\{y \in L^{2}(M) ; \operatorname{supp}(y) \subset M(\Gamma, h)\right\}$. In the same way, for $B \subset M^{\text {int }}$ an open set and $h: \bar{B} \rightarrow \mathbb{R}$ a piecewise continuous function satisfying $h>0$ pointwise, the set

$$
\left\{w_{H}(T, \cdot) ; H \in \mathcal{C}_{0}^{\infty}(\mathcal{B}(B, h ; T))\right\}
$$

is dense in $L^{2}(M(B, h))=\left\{y \in L^{2}(M) ; \operatorname{supp}(y) \subset M(B, h)\right\}$.

The $L^{2}$-topology used in the above lemma does not give control over the point values of $v_{\phi}$. For this reason, we need occasionally also the following lemma, that is analogous to Lemma 3.7 in [27].

Lemma 3. Let $T>0, \Gamma \subset \partial M, B \subset M^{\text {int }}$ and suppose that functions $h_{1}: \Gamma \rightarrow \mathbb{R}$ and $h_{2}: B \rightarrow \mathbb{R}$ are piecewise continuous. Let $x$ and $y$ be points in $M\left(\Gamma, h_{1}\right)^{\text {int }}$ and $M\left(B, h_{2}\right)^{\text {int }}$, respectively. Then there exist $\phi \in \mathcal{C}_{0}^{\infty}\left(\mathcal{B}\left(\Gamma, h_{1} ; T\right)\right)$ and $H \in \mathcal{C}_{0}^{\infty}\left(\mathcal{B}\left(B, h_{2} ; T\right)\right)$ such that the solution $v$ of 10 and the solution $w$ of $(11)$ satisfy $v(T, x) \neq 0$ and $w(T, y) \neq 0$.

\section{LOCAL DETERMINATION OF THE FIRST ORDER PERTURBATION}

In this section we prove Theorem 1. Before formulating the geometric step of our proof, that is, Proposition 1 below, let us introduce some notation. Let $\Gamma \subset \partial M$ be open. Then the boundary normal coordinates adapted to $\Gamma$ are given by the map

$$
(s, y) \mapsto \gamma(s ; y,-\nu), \quad y \in \Gamma, s \in\left[0, \sigma_{\Gamma, M}(y)\right),
$$


where the cut distance $\sigma_{\Gamma, M}: \Gamma \rightarrow(0, \infty)$ is defined by

$$
\begin{aligned}
\sigma_{\Gamma, M}(y) & =\max \left\{s \in\left(0, \tau_{M}(y)\right] ; d(\gamma(s ; y,-\nu), \Gamma)=s\right\}, \\
\tau_{M}(y) & =\sup \left\{s \in(0, \infty) ; \gamma(s ; y,-\nu) \in M^{\text {int }}\right\} .
\end{aligned}
$$

Here $\gamma(\cdot ; x, \xi)$ is the geodesic with the initial data $(x, \xi) \in T M$, and we recall that $\nu$ is the exterior unit normal on $\partial M$. We often write $\sigma_{\Gamma}=\sigma_{M, \Gamma}$. Note that $\sigma_{\Gamma}(y)>0$, see e.g. [21, p. 50].

We define

$$
M_{\Gamma}=\left\{\gamma(s ; y,-\nu) ; y \in \Gamma, s \in\left[0, \sigma_{\Gamma}(y)\right)\right\} .
$$

Then a point $x \in M_{\Gamma}$ is represented in the coordinates (15) by $(s, y)$, where $s=d(x, \Gamma)$ and $y$ is the unique closest point to $x$ in $\Gamma$.

We will also use the notations

$$
\begin{aligned}
B(p, r) & =\{x \in M ; d(x, p)<r\}, \quad p \in M, r>0, \\
B_{\partial M}(y, r) & =\{x \in \partial M ; d(x, y)<r\}, \quad y \in \partial M, r>0 .
\end{aligned}
$$

3.1. A convexity argument. Our aim is to construct a sequence of functions $\left(h_{k}\right)_{k \in \mathbb{N}}$ on $\mathcal{R}$ such that the difference of the domains of influences $M(\Gamma, s) \backslash M\left(\mathcal{R}, h_{k}\right)$ converges to a point $x \in M$ as $k \rightarrow \infty$. Here $\Gamma \subset \mathcal{R}$ and $s>0$ will be chosen suitably, and the point $x$ will lie in vicinity of $\mathcal{R}$. We will use this construction to enforce a sequence of solutions of (1) to converge, at a fixed time, to a point mass at $x$. The main result of this subsection can be stated as follows.

Proposition 1. Let $\Gamma \subset \partial M$ be open and strictly convex and let $\mathcal{K} \subset \Gamma$ be compact. Define for $p=(s, y) \in M_{\Gamma}$ and small $\epsilon>0$,

$$
C(p, \epsilon)=((s-\epsilon, s+\epsilon) \cap[0, \infty)) \times B_{\partial M}(y, \epsilon),
$$

in the coordinates (15). There exist a neighborhood $U \subset M_{\Gamma}$ of $\mathcal{K}$ such that for all $p \in U$ there is $\epsilon>0$ satisfying the following. For any $x \in C_{p}=C(p, \epsilon)$ there exists a sequence of functions $\left(h_{k, x}\right)_{k \in \mathbb{N}}$ in $C(\bar{\Gamma})$ such that the set

$$
X_{k, x}=M\left(B_{\partial M}(y, \epsilon), s+\epsilon\right)^{i n t} \backslash M\left(\Gamma, h_{k, x}\right), \quad k \in \mathbb{N},
$$

is a neighborhood of $x$, and diam $\left(X_{k, x}\right) \rightarrow 0$ as $k \rightarrow \infty$.

The functions $h_{k, x}$ are given explicitly by (21) below. In order to prove this result we will need three intermediate results.

Lemma 4. Let $\Gamma \subset \partial M$ be open and strictly convex, and let $\mathcal{K} \subset \Gamma$ be compact. Then there is $\delta(\mathcal{K})>0$ and a neighborhood $U(\mathcal{K}) \subset M_{\Gamma}$ of $\mathcal{K}$ such that, for all $x \in U(\mathcal{K})$ and $q \in B(x, \delta(\mathcal{K})) \backslash\{x\}$, there is $z \in \Gamma$ satisfying $d(z, q)<d(z, x)$. 
Proof. Let us consider a unit speed geodesic $\gamma(t)=(r(t), z(t))$ in coordinates (15) and denote the initial data of $\gamma$ by

$$
\gamma(0)=(s, y), \quad \dot{\gamma}(0)=(\rho, \eta)
$$

We will first show that there is a neighborhood $U \subset M$ of $\mathcal{K}$ and $\rho_{0}>0$ such that, for all $(s, y) \in \bar{U}$ and $\rho \in\left[-1, \rho_{0}\right]$, the geodesic $\gamma$ intersects $\Gamma$ and is distance minimizing until the intersection.

To this end recall that, in coordinates (15), the metric tensor $g$ is of the form

$$
g(s, y)=\left(\begin{array}{cc}
1 & 0 \\
0 & h(s, y)
\end{array}\right)
$$

We write $\left(x^{1}, \ldots, x^{n}\right)=(s, y)$ and $\partial_{j}=\partial_{x^{j}}$. Then it follows from (17) that the Christoffel symbols $\Gamma_{j k}^{l}$ satisfy for $\alpha, \beta=2, \ldots, n$,

$$
\Gamma_{\alpha 1}^{\beta}=\sum_{\kappa=2}^{n} \frac{1}{2} h^{\beta \kappa} \partial_{1} h_{\kappa \alpha}=-\sum_{\kappa=2}^{n} h^{\beta \kappa} \Gamma_{\alpha \kappa}^{1},
$$

and that the (scalar) second fundamental form of $\partial M$ satisfies

$$
I I\left(\partial_{\alpha}, \partial_{\beta}\right)(y)=-\sum_{\kappa=2}^{n} h_{\beta \kappa} \Gamma_{\alpha 1}^{\kappa}(0, y)=\Gamma_{\alpha \beta}^{1}(0, y) .
$$

The geodesic equations imply that

$$
r(t)=s+t \rho-\frac{t^{2}}{2} \sum_{\alpha, \beta=2}^{n} \Gamma_{\alpha \beta}^{1}(s, y) \eta^{\alpha} \eta^{\beta}+\mathcal{O}\left(t^{3}\right),
$$

see e.g. [34, p. 113]. Moreover, the strict convexity of $\Gamma$, the lower semi-continuity of the cut distance function $\sigma_{\Gamma}$ and the compactness of $\mathcal{K}$ imply that there is a neighborhood $U_{0} \subset M_{\Gamma}$ of $\mathcal{K}$ and $c>a>0$ such that, for all $(s, y) \in \overline{U_{0}}$,

$$
a|\eta|_{h}^{2} \leq \sum_{\alpha, \beta=2}^{n} \Gamma_{\alpha \beta}^{1}(s, y) \eta^{\alpha} \eta^{\beta} \leq c|\eta|_{h}^{2}
$$

We will consider only the case $|\eta|_{h}^{2}>1 / 2$. Note that if $\rho_{0}>0$ is small and $|\eta|_{h}^{2} \leq 1 / 2$, then $\rho<\rho_{0}$ implies that $\rho<0$ since $(\rho, \eta)$ is an unit vector. For small $t>0$, we have the bound

$$
s+t \rho-c t^{2} \leq r(t) \leq s+t \rho-\frac{a t^{2}}{8}
$$

The above formula implies that there is $\tau=\tau(s, y ; \rho, \nu)$ such that $r(\tau)=0$ and $r(t)>0$ for $t<\tau$. In addition, $\mathcal{K} \subset \Gamma$ is closed and $\gamma$ is unit speed. Thus there is $\sigma>0$ such that, for $s \leq \sigma, y \in \mathcal{K}, \rho \leq \sigma$ with 


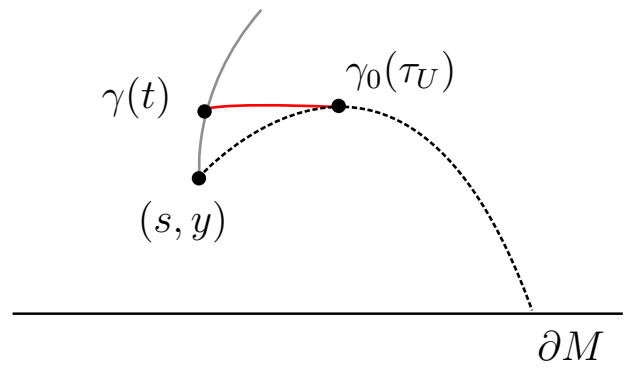

Figure 1. A schematic of the short cut argument in the proof of Lemma 4. The geodesics $\beta_{t}, \gamma$ and $\gamma_{0}$ are depicted by the solid red, solid grey and dashed black curves, respectively.

$(s, y) \in U_{0}$, the geodesic $\gamma(t)$ intersects $\Gamma$ at $t=\tau$. Moreover, $y(t) \in \Gamma$ for $0 \leq t \leq \tau$ and $\gamma(t)$ is the distance minimizing up to $z_{\gamma}=\gamma(\tau)$. Thus, for $t \in(0, \tau)$,

$$
d\left(z_{\gamma}, \gamma(t)\right)=\tau-t<d\left(z_{\gamma}, \gamma(0)\right) .
$$

Let us emphasize that the case $s=0$ is also allowed in the above argument. We take $U=\left\{(s, y) \in U_{0} ; s<\sigma\right\}$ and $\rho_{0}=\sigma$.

Let $(s, y) \in U,(\rho, \eta)$ be a unit vector and suppose that $\rho>\rho_{0}$. We may choose $\eta_{0}=b \eta, 0<b<1$, such that $\left(\rho_{0}, \eta_{0}\right)$ is also a unit vector at $(s, y)$. Then the geodesic $\gamma_{0}(s)$ with the initial data

$$
\gamma_{0}(0)=(s, y), \quad \dot{\gamma}_{0}(0)=\left(\rho_{0}, \eta_{0}\right)
$$

intersects $\Gamma$ at $z_{\gamma_{0}}$ and is distance minimizing until the intersection.

As $\rho_{0}>0$ we have that $\tau\left(s, y ; \rho_{0}, \eta\right)$ is strictly positive for $(s, y) \in \bar{U}$ and $\eta_{0} \in S:=\left\{\eta \in \mathbb{R}^{n-1} ;|\eta|_{h}^{2}+\rho_{0}^{2}=1\right\}$. Together with continuity of $\tau$ this implies

$$
\tau_{U}:=\min _{(s, y) \in \bar{U}, \eta_{0} \in S} \tau\left(s, y ; \rho_{0}, \eta_{0}\right) / 2>0 .
$$

Let $\beta_{t}:\left[0, l_{t}\right] \rightarrow M$ be the distance minimizing unit geodesic from $\gamma(t)$ to $\gamma_{0}\left(\tau_{U}\right)$, see Figure 1. The first variation formula, see e.g. [32, Prop. 10.2], implies that

$$
\left.\partial_{t} d\left(\gamma_{0}\left(\tau_{U}\right), \gamma(t)\right)\right|_{t=0}=\left.\partial_{t} \int_{0}^{l_{t}}\left|\dot{\beta}_{t}(r)\right| d r\right|_{t=0}=-\left.\left(\dot{\beta}_{t}(0), \partial_{t} \beta_{t}(0)\right)_{g}\right|_{t=0} .
$$

Observe that $\left.\dot{\beta}_{t}(0)\right|_{t=0}=\dot{\gamma}_{0}(0)$ and that $\partial_{t} \beta_{t}(0)=\dot{\gamma}(0)$. Hence

$$
\left.\partial_{t} d\left(\gamma_{0}\left(\tau_{U}\right), \gamma(t)\right)\right|_{t=0}=-\left(\dot{\gamma}_{0}(0), \dot{\gamma}(0)\right)_{g}=-\rho_{0} \rho-b\left|\eta_{0}\right|_{h}^{2} \leq-\rho_{0}^{2} .
$$

It follows from the above inequality together with the relative compactness of $U$ that there is $\delta>0$ such that, if $t \in(0, \delta),(s, y) \in U$, 
$\rho>\rho_{0}$, then

$$
d\left(z_{\gamma_{0}}, \gamma(t)\right) \leq d\left(z_{\gamma_{0}}, \gamma(0)\right)-t \rho_{0}^{2} / 2 .
$$

The claim now follows from (18) and $(19)$ by taking $\delta(\mathcal{K})=\min (\tau, \delta)$, $x=\gamma(0)$ and $q=\gamma(t)$ for $t \in(0, \delta(\mathcal{K}))$.

Lemma 5. Let $\Gamma \subset \partial M$ be open and let $x \in M_{\Gamma}$. Then, for all $q \in M(\Gamma, d(x, \Gamma)) \backslash\{x\}$, there is $z \in \bar{\Gamma}$ satisfying $d(z, q)<d(z, x)$.

Proof. Let $x=(s, y), s=d(x, \Gamma)$, in coordinates (15), and let $z$ be a closest point to $q$ in $\bar{\Gamma}$. If $z \neq y$ then

$$
d(z, q)=d(q, \Gamma) \leq d(x, \Gamma)<d(z, x),
$$

since $z$ is not the closest point to $x$ in $\Gamma$. Suppose now that $z=y$ and write $r=d(y, q)$. Then $r \leq d(x, \Gamma)=s$ and $q=(r, y)$ in coordinates (15). Moreover $q \neq x$, whence $r<s$.

Lemma 6. Let $\delta>0$ and let $p \in M_{\Gamma}$ have the boundary normal coordinates $(s, y)$. Then there is $\epsilon=\epsilon(p, \delta)>0$ such that for all $x \in B(p, \epsilon)$,

$$
M\left(B_{\partial M}(y, \epsilon), s+\epsilon\right) \subset M(\Gamma, d(x, \Gamma)) \cup B(x, \delta) .
$$

Proof. To prove (20) we assume the contrary. Then there exist sequences $\epsilon_{n} \rightarrow 0$,

$$
x_{n}=\left(r_{n}, z_{n}\right) \in B\left(p, \epsilon_{n}\right), \quad q_{n} \in M\left(B_{\partial M}\left(y, \epsilon_{n}\right), s+\epsilon_{n}\right),
$$

such that $d\left(q_{n}, \Gamma\right)>r_{n}$ and $d\left(q_{n}, x_{n}\right) \geq \delta$. Taking if necessary a subsequence, we may assume that $q_{n} \rightarrow q$. Then it follows from the above that

$$
d(q, y) \leq s, \quad d(q, \Gamma) \geq s, \quad d(q, p) \geq \delta .
$$

This is a contradiction since the first two conditions imply $q=p$.

Armed with these lemmas we are now in position to complete the proof of Proposition 1 .

Proof of Proposition 1. We assume that $\delta>0$ and $U \subset M_{\Gamma}$ are as in Lemma 4. Moreover, for $p=(s, y) \in U$ we fix $\epsilon=\epsilon(p, \delta)>0$ as in Lemma 6. We decrease $\epsilon>0$, if necessary, so that $B_{\partial M}(y, \epsilon) \subset \Gamma$ and that in the coordinates (15), $C(p, \epsilon) \subset B(p, \delta)$. Then, for any $x \in C(p, \epsilon)$ we set

$$
h_{k, x}(z)=d(z, x)-1 / k, \quad z \in \Gamma .
$$

The set $X_{k, x}$ is visualized in Figure 2. It is clear that $X_{k+1, x} \subset X_{k, x}$ and that $x \in X_{k, x}$ for all $k \in \mathbb{N}$. Suppose that $q \in \bar{X}_{k, x}$ for all $k \in \mathbb{N}$. If $q \notin B(x, \delta)$ then (20) yields that $q \in M(\Gamma, d(x, \Gamma))$. Now Lemma 

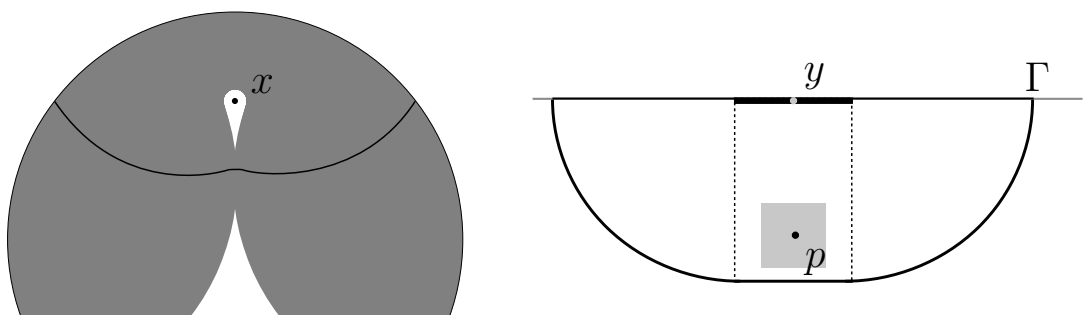

Figure 2. Left. A part of the domain of influence $M\left(\Gamma, h_{k, x}\right)$ in gray, where $(M, g)$ is the Euclidean unit disk, $x=(0,3 / 5)$, $k=20$, and $\Gamma$ is slightly less than the upper half circle. The black curve is the boundary of $M\left(B_{\partial M}(y, \epsilon), s+\epsilon\right)$ where $p=(s, y)$, $y=(0,1), s=1 / 2$ and $\epsilon=1 / 5$. The set $X_{k, x}$ is the white region around $x$. Right. Schematic diagram of the sets $B_{\partial M}(y, \epsilon) \subset \Gamma$, in black around the gray point $y$, and $C\left(p, \epsilon^{\prime}\right)$, in gray around the black point $p=(s, y)$. Here $\epsilon>\epsilon^{\prime}>0$. The black curve is the boundary of $M\left(B_{\partial M}(y, \epsilon), s+\epsilon\right)$.

5 implies that $q \in M\left(\Gamma, h_{k, x}\right)$ int for large $k$ which is a contradiction with $q \in \bar{X}_{k, x}$. If, however, $q \in B(x, \delta) \backslash\{x\}$, then Lemma 4 implies that $q \in M\left(\Gamma, h_{k, x}\right)$ int for large $k$ which is again a contradiction. Thus $q=x$. As the sequence of sets $X_{k, x}$ is decreasing and $\bigcap_{k \geq 1} \overline{X_{k, x}}=\{x\}$, we have that diam $\left(X_{k, x}\right) \rightarrow 0$ as $k \rightarrow \infty$.

3.2. Localized solutions. We denote by $|X|$ the Riemannian volume of a measurable set $X \subset M$. Also, we write

$$
\mathbb{U}_{A, q}: L^{2}((0, T) \times \mathcal{S}) \ni f \rightarrow u_{f}(T, \cdot) \in L^{2}(M),
$$

where $u_{f}$ is the solution of (1). If (1) is exactly controllable from $\mathcal{S}$ in time $T$, then $\mathbb{U}_{A, q}$ is a continuous surjective linear map from $L^{2}((0, T) \times \mathcal{S})$ to $L^{2}(M)$. In this case, using the fact that $L^{2}((0, T) \times$ $\mathcal{S})$ is a Hilbert space, we can consider the restriction $\mathbb{U}_{1}$ of $\mathbb{U}_{A, q}$ to $\mathbb{U}_{A, q}^{-1}(\{0\})^{\perp}$, where $\mathbb{U}_{A, q}^{-1}(\{0\})^{\perp}$ denotes the orthogonal complement of $\mathbb{U}_{A, q}^{-1}(\{0\})$ in $L^{2}((0, T) \times \mathcal{S})$. It is clear that $\mathbb{U}_{1}$ is continuous and oneto-one, from $\mathbb{U}_{A, q}^{-1}(\{0\})^{\perp}$ to $L^{2}(M)$, and, since both $\mathbb{U}_{A, q}^{-1}(\{0\})^{\perp}$ and $L^{2}(M)$ are Hilbert spaces, applying [8, Corollary 2.7, pp. 35] (see also [14, pp. 33-34]) we deduce that $\mathbb{U}_{1}$ is an isomorphism from $\mathbb{U}_{A, q}^{-1}(\{0\})^{\perp}$ to $L^{2}(M)$. Fixing $\mathbb{U}_{A, q}^{\dagger}=\mathbb{U}_{1}^{-1}$ we obtain a continuous linear map from $L^{2}(M)$ to $L^{2}((0, T) \times \mathcal{S})$ such that $\mathbb{U}_{A, q} \mathbb{U}_{A, q}^{\dagger}=\mathbb{U}_{1} \mathbb{U}_{1}^{-1}=I d$. 
In this subsection we will prove the following proposition that, together with Proposition 1, will allow us to enforce a sequence of solutions of (1) to converge, at a fixed time, to a point mass.

Proposition 2. Let $\mathcal{X} \subset M$ be open, $x \in \mathcal{X}$ and let $X_{k} \subset M, k \in \mathbb{N}$, be a sequence of neighborhoods of $x$ satisfying $\lim _{k \rightarrow \infty} \operatorname{diam}\left(X_{k}\right)=0$. Let $\psi_{0} \in C_{0}^{\infty}(\mathcal{X})$ satisfy $\psi_{0}(x) \neq 0$. Let $T>0$ and suppose that a sequence $\left(f_{k}\right)_{k \in \mathbb{N}}$ of functions in $L^{2}((0, T) \times \mathcal{S})$ satisfies

(i) there is $C>0$ such that $\left\|f_{k}\right\|_{L^{2}((0, T) \times \mathcal{S})} \leq C\left|X_{k}\right|^{-1 / 2}$ for all $k \in \mathbb{N}$,

(ii) $\operatorname{supp}\left(u_{f_{k}}(T, \cdot)\right) \subset \bar{X}_{k} \cup(M \backslash \mathcal{X})$ for all $k \in \mathbb{N}$,

(iii) $\left(\left\langle u_{f_{k}}(T, \cdot), \psi_{0}\right\rangle_{L^{2}(M)}\right)_{k \in \mathbb{N}}$ converges.

Then there is $\kappa \in \mathbb{C}$ such that $\left\langle u_{f_{k}}(T, \cdot), \psi\right\rangle_{L^{2}(M)} \rightarrow \kappa \psi(x)$ for all functions $\psi \in C_{0}^{\infty}(\mathcal{X})$.

Furthermore, if the wave equation (1) is exactly controllable from $\mathcal{S}$ in time $T$, then the sequence $f_{k}=\mathbb{U}_{A, q}^{\dagger} 1_{X_{k}} /\left|X_{k}\right|, k \in \mathbb{N}$, satisfies (i)-(iii), and the corresponding $\kappa$ is 1 .

Let us emphasize that $\mathcal{X}$ is open in the topology of $M$, a manifold with boundary. In particular, $\mathcal{X}$ may intersect $\partial M$ in which case $x$ may belong to $\partial M$.

Proof. Let $\psi \in C_{0}^{\infty}(\mathcal{X})$. Observe that $\operatorname{supp}\left(u_{f_{k}}(T, \cdot) \psi\right) \subset \bar{X}_{k}$. Fixing

$$
R_{k}(\psi)=\left\langle u_{f_{k}}(T, \cdot), \psi\right\rangle_{L^{2}(M)}-\psi(x) \int_{X_{k}} \overline{u_{f_{k}}(T, x)} d V_{g}(x)
$$

where $d V_{g}$ denotes the Riemannian volume, we get

$$
\left\langle u_{f_{k}}(T, \cdot), \psi\right\rangle_{L^{2}(M)}=\psi(x)\left\langle u_{f_{k}}(T, \cdot), 1\right\rangle_{L^{2}\left(X_{k}\right)}+R_{k}(\psi) .
$$

Using some local coordinates $\widetilde{x}$ in $X_{k}$ for all large enough $k$, the remainder term satisfies

$$
\begin{aligned}
\left|R_{k}(\psi)\right| & \leq \int_{X_{k}}\left|u_{f_{k}}(T, \widetilde{x}) \| \psi(\widetilde{x})-\psi(x)\right| d V_{g}(\widetilde{x}) \\
& \leq C\|d \psi\|_{C\left(X_{k}\right)} \int_{X_{k}}\left|u_{f_{k}}(T, \widetilde{x})\right| d(\widetilde{x}, x) d V_{g}(\widetilde{x}) \\
& \leq C\|d \psi\|_{C\left(X_{k}\right)}\left\|u_{f_{k}}(T, \cdot)\right\|_{L^{2}(M)}\left(\int_{X_{k}} d^{2}(\widetilde{x}, x) d V_{g}(\widetilde{x})\right)^{1 / 2} \\
& \leq C\|d \psi\|_{C(M)} \operatorname{diam}\left(X_{k}\right) \rightarrow 0 .
\end{aligned}
$$

Notice that the constant $C>0$ may increase between the inequalities and that, at the last inequality, we use

$$
\left\|u_{f_{k}}(T, \cdot)\right\|_{L^{2}(M)} \leq C\left\|f_{k}\right\|_{L^{2}((0, T) \times \mathcal{S})},
$$


see [29], together with (i). We choose $\psi=\psi_{0}$ and see that the limit

$$
\lim _{k \rightarrow \infty}\left\langle u_{f_{k}}(T, \cdot), 1\right\rangle_{L^{2}\left(X_{k}\right)}=\frac{1}{\psi_{0}(x)} \lim _{k \rightarrow \infty}\left(\left\langle u_{f_{k}}(T, \cdot), \psi_{0}\right\rangle_{L^{2}(M)}-R_{k}\left(\psi_{0}\right)\right)
$$

exists. We denote the limit by $\kappa$. Thus for any $\psi \in C_{0}^{\infty}(\mathcal{X})$ it holds that $\left\langle u_{f_{k}}(T, \cdot), \psi\right\rangle_{L^{2}(M)} \rightarrow \kappa \psi(x)$ as $k \rightarrow \infty$.

Finally, it is clear that $f_{k}=\mathbb{U}_{A, q}^{\dagger} 1_{X_{k}} /\left|X_{k}\right|$ has the properties (i)-(iii) with $\kappa=1$.

3.3. Local recovery near the set $\mathcal{R}$. Armed with the localization procedure given by Propositions 1 and 2 , we prove Theorem 1 in this section.

From now on, we fix $A_{j} \in C^{\infty}(M ; T M), q_{j} \in \mathcal{C}^{\infty}(M ; \mathbb{C}), j=1,2$, and, for functions $f \in C_{0}^{\infty}((0,+\infty) \times \partial M), \phi \in C_{0}^{\infty}((0,+\infty) \times \partial M)$ and $H \in C_{0}^{\infty}((0,+\infty) \times B)$, we consider $u_{j, f}, v_{j, \phi}, w_{j, H}$ solving respectively (1), (10), (11) with $A=A_{j}$ and $q=q_{j}$. We write also

$$
\mathcal{A}_{j}=\Delta_{g}-A_{j}-q_{j}, \quad \mathcal{A}_{j}^{*}=\Delta_{g}+\overline{A_{j}}-\left(\overline{q_{j}}-\operatorname{div}_{g}\left(\overline{A_{j}}\right)\right) .
$$

Before proving Theorem 1 we still need to establish two lemmas.

Lemma 7. Let $\Gamma \subset \partial M$ and $B \subset M^{\text {int }}$ be open. Let $T>0$ and $h: \bar{\Gamma} \rightarrow[0, T]$ be piecewise continuous. Let $\mathcal{C} \subset M(\Gamma, h) \cap M^{\text {int }}$ be open and let $\kappa \in C^{\infty}(\mathcal{C})$ be nowhere vanishing. Then the condition

$$
v_{1, \phi}(T, x)=\kappa v_{2, \phi}(T, x), \quad \phi \in C_{0}^{\infty}(\mathcal{B}(\Gamma, h ; T)), x \in \mathcal{C},
$$

implies that $\mathcal{A}_{1}=\bar{\kappa}^{-1} \mathcal{A}_{2} \bar{\kappa}$ on $\mathcal{C}$. In the same way, for $h: \bar{\Gamma} \rightarrow[0, T]$ piecewise continuous, $\mathcal{C} \subset M(B, h) \cap M^{\text {int }}$ and $\kappa \in C^{\infty}(\mathcal{C})$ be nowhere vanishing, the condition

$$
w_{1, \kappa H}(T, x)=\kappa w_{2, H}(T, x), \quad H \in C_{0}^{\infty}(\mathcal{B}(B, h ; T)), x \in \mathcal{C},
$$

implies that $\mathcal{A}_{1}=\bar{\kappa}^{-1} \mathcal{A}_{2} \bar{\kappa}$ on $\mathcal{C}$.

Proof. Since the proof of these two results are similar, we will only show that (22) implies $\mathcal{A}_{1}=\bar{\kappa}^{-1} \mathcal{A}_{2} \bar{\kappa}$ on $\mathcal{C}$. We start by proving that (22) implies

$$
\mathcal{A}_{1}^{*} v_{1, \phi}(T, \cdot)=\kappa \mathcal{A}_{2}^{*} v_{2, \phi}(T, \cdot), \quad \phi \in C_{0}^{\infty}(\mathcal{B}(\Gamma, h ; T)) .
$$

For this purpose, we fix $\phi \in C_{0}^{\infty}(\mathcal{B}(\Gamma, h ; T))$ and remark that there is $\varepsilon>0$ such that $\operatorname{supp}(\phi) \subset[\epsilon,+\infty) \times \partial M$ and $\phi \in C_{0}^{\infty}(\mathcal{B}(\Gamma, h-\epsilon ; T))$. Thus, taking into account the translation invariance in time of $(10)$ and fixing $\phi_{s}:(t, x) \mapsto \phi(s+t, x)$, we obtain that

$$
v_{j, \phi_{s}}(T, \cdot)=v_{j, \phi}(s+T, \cdot), \quad s \in[0, \epsilon), j=1,2
$$


and (22) implies

$$
v_{1, \phi}(s+T, \cdot)=\kappa v_{2, \phi}(s+T, \cdot), \quad s \in[0, \epsilon) .
$$

Differentiating twice this identity with respect to $s$, we get (24).

Now let $\psi$ be a function in $C_{0}^{\infty}(\mathcal{C})$. Applying again $(22)$, we can compute

$$
\begin{aligned}
\left\langle\kappa \mathcal{A}_{2}^{*} v_{2, \phi}(T, \cdot), \psi\right\rangle_{L^{2}(\mathcal{C})} & =\left\langle\kappa v_{2, \phi}(T, \cdot), \bar{\kappa}^{-1} \mathcal{A}_{2} \bar{\kappa} \psi\right\rangle_{L^{2}(\mathcal{C})} \\
& =\left\langle v_{1, \phi}(T, \cdot), \bar{\kappa}^{-1} \mathcal{A}_{2} \bar{\kappa} \psi\right\rangle_{L^{2}(\mathcal{C})}
\end{aligned}
$$

Applying (24), we get

$$
\left\langle\mathcal{A}_{1}^{*} v_{1, \phi}(T, \cdot), \psi\right\rangle_{L^{2}(\mathcal{C})}=\left\langle\kappa \mathcal{A}_{2}^{*} v_{2, \phi}(T, \cdot), \psi\right\rangle_{L^{2}(\mathcal{C})}=\left\langle v_{1, \phi}(T, \cdot), \bar{\kappa}^{-1} \mathcal{A}_{2} \bar{\kappa} \psi\right\rangle_{L^{2}(\mathcal{C})}
$$

and it follows

$$
\left\langle v_{1, \phi}(T, \cdot),\left(\mathcal{A}_{1}-\bar{\kappa}^{-1} \mathcal{A}_{2} \bar{\kappa}\right) \psi\right\rangle_{L^{2}(\mathcal{C})}=0, \quad \psi \in \mathcal{C}_{0}^{\infty}(\mathcal{C}) .
$$

As the functions $\left.v_{1, \phi}(T, \cdot)\right|_{\mathcal{C}}, \phi \in C_{0}^{\infty}(\mathcal{B}(\Gamma, h ; T))$, are dense on $L^{2}(\mathcal{C})$, we deduce from (25) that $\mathcal{A}_{1}=\bar{\kappa}^{-1} \mathcal{A}_{2} \bar{\kappa}$ on $\mathcal{C}$, which completes the proof of the lemma.

The next lemma will be used only for $j=2$.

Lemma 8. Let $\Gamma \subset \partial M$ and $B \subset M^{\text {int }}$ be open, and let $\mathcal{V}=\Gamma$ or $\mathcal{V}=B$. Let $T>0$ and let $h: \overline{\mathcal{V}} \rightarrow[0, T]$ be piecewise continuous. In the case when $\mathcal{V}=B$ suppose, moreover, that $h>0$ pointwise. Let $\mathcal{C}_{1} \subset M(\Gamma, h) \cap M^{\text {int }}$ and $\mathcal{C}_{2} \subset M(B, h) \cap M^{\text {int }}$ be open and let $\kappa_{\ell}: \mathcal{C}_{\ell} \rightarrow \mathbb{C}$. Then the following properties hold:

(1) For $j=1,2$, if $\kappa_{1} v_{j, \phi}(T, \cdot) \in C^{\infty}\left(\mathcal{C}_{1}\right)$ for all $\phi \in C_{0}^{\infty}(\mathcal{B}(\Gamma, h ; T))$ then $\kappa_{1} \in C^{\infty}\left(\mathcal{C}_{1}\right)$. In the same way, if $\kappa_{2} w_{j, H}(T, \cdot) \in C^{\infty}\left(\mathcal{C}_{2}\right)$ for all $H \in C_{0}^{\infty}(\mathcal{B}(B, h ; T))$ then $\kappa_{2} \in C^{\infty}\left(\mathcal{C}_{2}\right)$.

(2) If for all $x \in \mathcal{C}_{1}$ there is $\phi \in C_{0}^{\infty}(\mathcal{B}(\Gamma, h ; T))$ such that

$$
\kappa_{1}(x) v_{j, \phi}(T, x) \neq 0
$$

then $\kappa_{1}(x) \neq 0$ for all $x \in \mathcal{C}_{1}$. If for all $x \in \mathcal{C}_{2}$ there is $H \in$ $C_{0}^{\infty}(\mathcal{B}(B, h ; T))$ such that $\kappa_{2}(x) w_{j, H}(T, x) \neq 0$ then $\kappa_{2}(x) \neq 0$ for all $x \in \mathcal{C}_{2}$.

Moreover, in the case $\mathcal{V}=\Gamma$ we can enforce smoothness up to the boundary, that is, we define $\widetilde{\mathcal{C}}=\mathcal{C}_{1} \cup\left(S \cap \overline{\mathcal{C}}_{1}\right)$ where $S$ is an open set in $\partial M$ such that $h>0$ in $S$, and have the following:

(3) For $j=1,2$, if $\kappa_{1} v_{j, \phi}(T, \cdot) \in C^{\infty}(\widetilde{\mathcal{C}})$ for all $\phi \in C_{0}^{\infty}(\mathcal{B}(\Gamma, h ; T))$ then $\kappa_{1} \in C^{\infty}(\widetilde{\mathcal{C}})$. 
Proof. The proof for $\mathcal{V}=\Gamma$ or $\mathcal{V}=B$ being similar, we consider only the results for $\mathcal{V}=\Gamma$. Let $x \in \mathcal{C}_{1}$. By Lemma3, there is a neighborhood $U$ of $x$ and $\phi \in C_{0}^{\infty}(\mathcal{B}(\Gamma, h ; T))$ such that $v_{j, \phi}(T, \cdot)$ is non-vanishing in $U$. We have that $\kappa_{1}=\frac{\kappa_{1} v_{j, \phi}(T, \cdot)}{v_{j, \phi}(T, \cdot)}$ in $U$, and (1) and (2) follow.

Suppose now that $x \in S \cap \overline{\mathcal{C}}_{1}$. Then there is a neighborhood of $U \subset$ $M$ of $x$ and $\phi \in C_{0}^{\infty}(\mathcal{B}(\Gamma, h ; T))$ such that $v_{j, \phi}(T, \cdot)$ is non-vanishing in $U$, since on $S$ we can choose $v_{j, \phi}(T, \cdot)=\phi(T, \cdot)$ to be non-vanishing. The function $v_{j, \phi}(T, \cdot)$ is smooth up to $\partial M$, whence $\kappa_{1}$ is smooth in $U$.

We are ready to prove the local result formulated in the introduction.

Proof of Theorem 1. As the wave equation is exactly controllable from $\mathcal{S}$ in time $T$, [3, Theorem 3.2] implies that $\sigma_{\mathcal{R}} \leq T$ pointwise on $\mathcal{R}$. We recall that $\sigma_{\mathcal{R}}$ is defined by $(16)$. For $j=1,2$ and $\kappa \in C^{\infty}\left(\overline{\Omega_{s}}\right)$, we fix $K_{j, \mathcal{R}}^{T}$ given by (8) for $\Lambda_{\Gamma}=\Lambda_{j, S, \mathcal{R}}^{2 T}$ and applying (4) we deduce that

$$
K_{1, \mathcal{R}}^{T}=K_{2, \mathcal{R}}^{T} \text {. }
$$

Let $\mathcal{K} \subset \mathcal{R}$ be compact, and consider the sets defined in Proposition 1 for $\Gamma=\mathcal{R}, U \subset M_{\mathcal{R}}$ and $p=(s, y) \in U$. We write $C_{p}(\mathcal{K})=C_{p}$ to emphasize the dependence on $\mathcal{K}$, and use an analogous notation also for other quantities in Proposition 1. We will start by proving that there exists $\kappa \in C^{\infty}\left(C_{p}(\mathcal{K})\right)$ such that the following identity holds

$$
v_{1, \phi}(T, x)=\kappa(x) v_{2, \phi}(T, x),
$$

for $\phi \in C_{0}^{\infty}\left(\mathcal{B}\left(B_{\partial M}(y, \epsilon), s+\epsilon ; T\right)\right)$ and $x \in C_{p}(\mathcal{K})$. For any $x \in$ $C_{p}(\mathcal{K})$, we consider functions $h_{k, x}$ and sets $X_{k, x}$ satisfying the properties described in Proposition 1. We will apply the result of Proposition 2, with $\mathcal{X}=M\left(B_{\partial M}(y, \epsilon), s+\epsilon\right)^{\text {int }}$ and $X_{k}=X_{k, x}$. Using the exact controllability assumption, we fix

$$
f_{k, x}=\mathbb{U}_{A_{1}, q_{1}}^{\dagger} 1_{X_{k, x}} /\left|X_{k, x}\right|, \quad k \in \mathbb{N} .
$$

We remark that

$$
\lim _{k \rightarrow+\infty}\left\langle u_{1, f_{k, x}}(T, \cdot), \psi\right\rangle_{L^{2}(M)}=\psi(x), \quad \psi \in C_{0}^{\infty}(\mathcal{X}) .
$$

Let us now show that the conditions (i)-(iii) of Proposition 2 are fulfilled with respect to $j=2$. Clearly (i) holds, as it does not depend on $j=1,2$. The equations (12) and (26) imply that

$$
\begin{aligned}
\left\langle u_{1, f_{k, x}}(T, \cdot), v_{1, \phi}(T, \cdot)\right\rangle_{L^{2}(M)} & =\left\langle K_{1, \mathcal{R}}^{T} f_{k, x}, \phi\right\rangle_{L^{2}((0, T) \times \mathcal{R})} \\
& =\left\langle K_{2, \mathcal{R}}^{T} f_{k, x}, \phi\right\rangle_{L^{2}((0, T) \times \mathcal{R})} \\
& =\left\langle u_{2, f_{k, x}}(T, \cdot), v_{2, \phi}(T, \cdot)\right\rangle_{L^{2}(M)} .
\end{aligned}
$$


Finite speed of propagation implies that $\operatorname{supp}\left(v_{1, \phi}(T, \cdot)\right) \subset M\left(\Gamma, h_{k, x}\right)$ for all $\phi \in C_{0}^{\infty}\left(\mathcal{B}\left(\mathcal{R}, h_{k, x} ; T\right)\right)$. Observe that $u_{1, f_{k, x}}(T, \cdot)=1_{X_{k, x}} /\left|X_{k, x}\right|$ by $(28)$, and recall that by definition $\mathcal{X}=M\left(B_{\partial M}(y, \epsilon), s+\epsilon\right)^{\text {int }}$ and $X_{k, x}=\mathcal{X} \backslash M\left(\Gamma, h_{k, x}\right)$, see Proposition 1 for the latter. Hence

$$
\left\langle u_{2, f_{k, x}}(T, \cdot), v_{2, \phi}(T, \cdot)\right\rangle_{L^{2}(M)}=\left\langle u_{1, f_{k, x}}(T, \cdot), v_{1, \phi}(T, \cdot)\right\rangle_{L^{2}(M)}=0
$$

and the density result of Lemma 2 implies that $u_{2, f_{k, x}}(T, \cdot)=0$ in $M\left(\Gamma, h_{k, x}\right)$. Therefore

$$
\operatorname{supp}\left(u_{2, f_{k, x}}(T, \cdot)\right) \subset M \backslash M\left(\Gamma, h_{k, x}\right) \subset X_{k, x} \cup(M \backslash \mathcal{X}),
$$

and (ii) holds. Moreover, equations (29)-(30) imply that the sequence

$$
\left(\left\langle u_{2, f_{k, x}}(T, \cdot), v_{2, \phi}(T, \cdot)\right\rangle_{L^{2}(M)}\right)_{k \in \mathbb{N}}
$$

converges for any $\phi \in C_{0}^{\infty}\left(\mathcal{B}\left(B_{\partial M}(y, \epsilon), s+\epsilon ; T\right)\right)$. Thus, condition (iii) of Proposition 2 is also fulfilled. Note that by Lemma 3 , the function $\phi$ can be chosen so that $v_{2, \phi}(T, x) \neq 0$. According to Proposition 2 there exists $\kappa_{x}$ such that

$$
\lim _{k \rightarrow+\infty}\left\langle u_{2, f_{k, x}}(T, \cdot), \psi\right\rangle_{L^{2}(M)}=\kappa_{x} \psi(x), \quad \psi \in C_{0}^{\infty}(\mathcal{X}) .
$$

We define a function $\kappa: C_{p}(\mathcal{K}) \rightarrow \mathbb{C}$ by $\kappa(x)=\kappa_{x}$, and remark that applying (29) with $\psi=v_{1, \phi}(T, \cdot)$ for $\phi \in C_{0}^{\infty}\left(\left(B_{\partial M}(y, \epsilon), s+\epsilon, T\right)\right)$, and using (30), we have

$$
v_{1, \phi}(T, x)=\lim _{k \rightarrow+\infty}\left\langle u_{2, f_{k, x}}(T, \cdot), v_{2, \phi}(T, \cdot)\right\rangle_{L^{2}(M)}=\kappa(x) v_{2, \phi}(T, x) .
$$

This establishes (27). Moreover, combining (27) with the fact that $v_{1, \phi}(T, \cdot) \in C^{\infty}(M)$, for $\phi \in C_{0}^{\infty}\left(\mathcal{B}\left(B_{\partial M}(y, \epsilon), s+\epsilon, T\right)\right)$, and applying Lemma 8, we deduce that $\kappa \in C^{\infty}\left(C_{p}(\mathcal{K}) \cup\left(\overline{C_{p}(\mathcal{K})} \cap \mathcal{R}\right)\right)$. Finally, applying Lemma 3 we deduce that for all $x \in C_{p}(\mathcal{K})$ there exists $\phi_{x}$ such that $v_{\phi_{x}}(T, x) \neq 0$. Thus, 32 and Lemma 8 imply $\kappa$ is nowhere vanishing in $C_{p}(\mathcal{K})$.

We consider now a collection $\left\{\mathcal{K}_{i} ; i \in I\right\}$ of compact sets in $\mathcal{R}$ and $p_{i}=\left(s_{i}, y_{i}\right)$ lying in a neighborhood of $\mathcal{K}_{i}$ as in Proposition 1 . We assume that $\left\{\mathcal{K}_{i} ; i \in I\right\}$ and $\left\{p_{i} ; i \in I\right\}$ are chosen in such a way that $\bigcup_{i \in I} C_{p_{i}}\left(\mathcal{K}_{i}\right)$ is a neighborhood of $\mathcal{R}$. Repeating the above argumentation, for all $i \in I$, we find $\kappa_{i} \in C^{\infty}\left(C_{p_{i}}\left(\mathcal{K}_{i}\right) \cup\left(\overline{C_{p_{i}}\left(\mathcal{K}_{i}\right)} \cap \mathcal{R}\right)\right)$ such that, for all $x \in C_{p_{i}}\left(\mathcal{K}_{i}\right)$, we have

$$
\left.v_{1, \phi}(T, x)=\kappa_{i}(x) v_{2, \phi}(T, x), \quad \phi \in C_{0}^{\infty}\left(B_{\partial M}\left(y_{i}, \epsilon_{i}\right), s_{i}+\epsilon_{i}, T\right)\right) .
$$

Now let $i_{1}, i_{2} \in I$ be such that $C_{p_{i_{1}}}\left(\mathcal{K}_{i_{1}}\right) \cap C_{p_{i_{2}}}\left(\mathcal{K}_{i_{2}}\right) \neq \emptyset$. Since both $C_{p_{i_{1}}}\left(\mathcal{K}_{i_{1}}\right)$ and $C_{p_{i_{2}}}\left(\mathcal{K}_{i_{2}}\right)$ are cylindrical domains, $C_{p_{i_{1}}}\left(\mathcal{K}_{i_{1}}\right) \cap C_{p_{i_{2}}}\left(\mathcal{K}_{i_{2}}\right)$ 
is also cylindrical and we have $B_{\partial M}\left(y_{i_{1}}, \epsilon_{i_{1}}\right) \cap B_{\partial M}\left(y_{i_{2}}, \epsilon_{i_{2}}\right) \neq \emptyset$. Combining this with (33), we obtain for any

$$
\phi \in C_{0}^{\infty}\left(\mathcal{B}\left(B_{\partial M}\left(y_{i_{1}}, \epsilon_{i_{1}}\right) \cap B_{\partial M}\left(y_{i_{2}}, \epsilon_{i_{2}}\right), \max \left(s_{i_{1}}+\epsilon_{i_{1}}, s_{i_{2}}+\epsilon_{i_{2}}\right), T\right)\right)
$$

and any $x \in C_{p_{i_{1}}}\left(\mathcal{K}_{i_{1}}\right) \cap C_{p_{i_{2}}}\left(\mathcal{K}_{i_{2}}\right)$, the equation

$$
\kappa_{i_{2}}(x) v_{2, \phi}(T, x)=v_{1, \phi}(T, x)=\kappa_{i_{1}}(x) v_{2, \phi}(T, x) .
$$

In view of Lemma 3 , for any $x \in C_{p_{i_{1}}}\left(\mathcal{K}_{i_{1}}\right) \cap C_{p_{i_{2}}}\left(\mathcal{K}_{i_{2}}\right)$, we can choose $\phi$ such that $v_{2, \phi}(T, x) \neq 0$. Combining this with (34), we have

$$
\kappa_{i_{1}}(x)=\kappa_{i_{2}}(x), \quad x \in C_{p_{i_{1}}}\left(\mathcal{K}_{i_{1}}\right) \cap C_{p_{i_{2}}}\left(\mathcal{K}_{i_{2}}\right) .
$$

Therefore, we can define $\kappa \in C^{\infty}\left(\bigcup_{i \in I} C_{p_{i}}\left(\mathcal{K}_{i}\right)\right)$ such that, for all $i \in I$, $\kappa_{\mid C_{p_{i}}\left(\mathcal{K}_{i}\right)}=\kappa_{i}$. In light of $(33)$, we deduce that $\kappa_{\mid \mathcal{R}}=1$ and Lemma 8 implies that $\kappa \neq 0$. Moreover, applying Lemma 7 on $C_{p_{i}}\left(\mathcal{K}_{i}\right)$, for any $i \in I$, we deduce that

$$
\mathcal{A}_{1}=\kappa^{-1} \mathcal{A}_{2} \kappa=\Delta_{g}-\left(A_{2}+2 \kappa^{-1} \operatorname{grad}_{g} \kappa\right)-\left(q_{2}+\kappa\left(A_{2}-\Delta_{g}\right) \kappa^{-1}\right)
$$

holds true on $\bigcup_{i \in I} C_{p_{i}}\left(\mathcal{K}_{i}\right)$. Therefore, we can define $\mathcal{U}$ a neighborhood of $\mathcal{R}$, contained into $\bigcup_{i \in I} C_{p_{i}}\left(\mathcal{K}_{i}\right)$, such that $\kappa \in C^{\infty}(\overline{\mathcal{U}})$ and $\left(\left.A_{1}\right|_{\mathcal{U}},\left.q_{1}\right|_{\mathcal{U}}\right) \in \mathcal{G}_{\mathcal{U}, \mathcal{R}}\left(A_{2}, q_{2}\right)$. This completes the proof of the theorem.

\section{ReCOnstruction of the First ORDER PERTURBATION ALONG A CONVEX FOLIATION}

In this section we prove the global result stated in Theorem 2, The proof of this result is based on iterating the local reconstruction method of the previous section along the convex foliation.

4.1. Local recovery near the set $\Sigma_{s}$. Let $\Sigma_{s}, s \in(0,1]$, be a convex foliation satisfying (F1)-(F7). Let $\Gamma \subset \Sigma_{s}$ be open and let $h: \bar{\Gamma} \rightarrow \mathbb{R}$ be piecewise continuous. We recall that $M_{s}$ is defined in (F4), and consider the domain of influence on $M_{s}$,

$$
M_{s}(\Gamma, h):=\left\{x \in M_{s} ; \inf _{y \in \Gamma}\left(d_{M_{s}}(x, y)-h(y)\right) \leq 0\right\} .
$$

Here $d_{M_{s}}(x, y)$ is the distance function on $\left(M_{s}, g\right)$. We will also use the notation $d_{\bar{\Omega}_{s}}(x, y)$ for the distance function on $\left(\bar{\Omega}_{s}, g\right)$.

Lemma 9. Let $\Sigma_{s}, s \in(0,1]$, be a convex foliation satisfying (F1)$\left(F^{r}\right)$, and let $s \in(0,1]$. Let $h: \bar{\Sigma}_{s} \rightarrow \mathbb{R}$ be piecewise continuous. Then

$$
M_{s}\left(\Sigma_{s}, h\right) \cup \bar{\Omega}_{s}=M\left(\Omega_{s}, \widetilde{h}\right),
$$

where $\widetilde{h}(y)=\max \left(\sup _{z \in \Sigma_{s}}\left(h(z)-d_{\bar{\Omega}_{s}}(z, y)\right), d_{\bar{\Omega}_{s}}\left(y, \partial \Omega_{s}\right)\right)$. 
Proof. Let us show first that

$$
d(x, z)=d_{M_{s}}(x, z), \quad x, z \in M_{s} .
$$

It is enough to show that a shortest path $\gamma$ between $x$ and $z$ stays in $M_{s}$. To get a contradiction suppose that $S<s$, where

$$
S=\inf \left\{r \in[0, s] ; \gamma \cap \Sigma_{r} \neq \emptyset\right\},
$$

and we have used the notation $\Sigma_{0}=\mathcal{R}_{0}$. Let $p \in \gamma \cap \Sigma_{S}$. Let us consider first the case $S>0$. Then $\gamma$ is a geodesic near $p$. As $\gamma \cap \Omega_{S}=\emptyset$, the intersection is tangential. But then the strict convexity of $\Sigma_{S}$ implies that $\gamma$ is in $\Omega_{S}$ near $p$, which is a contradiction. On the other hand, if $S=0$ then the intersection must be tangential again, since a shortest path is $C^{1}$, see [1]. But this is impossible by the strict convexity of $\Sigma_{0} \subset \mathcal{R}$.

Let us now show 36 . Note that $\tilde{h}(y) \geq h(y)$ for $y \in \Sigma_{s}$ and that $\widetilde{h}>0$ on $\Omega_{s}$. Hence $M_{s}\left(\Sigma_{s}, h\right) \cup \bar{\Omega}_{s} \subset M\left(\Omega_{s}, \widetilde{h}\right)$. On the other hand, if $x \in M\left(\Omega_{s}, \widetilde{h}\right) \backslash \bar{\Omega}_{s}$ then there is $y \in \bar{\Omega}_{s}$ such that $d(x, y)-\widetilde{h}(y) \leq 0$ and $z \in \bar{\Sigma}_{s}$ such that $\widetilde{h}(y)=h(z)-d_{\bar{\Omega}_{s}}(z, y)$. Thus

$$
d_{M_{s}}(x, z)-h(z)=d(x, z)-d_{\bar{\Omega}_{s}}(z, y)-\widetilde{h}(y) \leq d(x, y)-\widetilde{h}(y) \leq 0,
$$

and $x \in M_{s}\left(\Sigma_{s}, h\right)$.

Let us prove next the following analogue of Theorem 1 with internal data on $\Omega_{s}$. Note that contrary to Theorem 1 , we do not require $\kappa$ to have a specific value on $\Sigma_{s}$. We recall that for and open set $U \subset M^{\text {int }}$ and $f \in C_{0}^{\infty}((0, \infty) \times \mathcal{S})$,

$$
\mathcal{T}_{j, U, \kappa} f=\left.\kappa u_{j}\right|_{(0, \infty) \times U},
$$

where $u_{j}$ is the solution of (1) for $A=A_{j}$ and $q=q_{j}$.

Lemma 10. Let $\mathcal{S} \subset \partial M$ be open and suppose that the wave equation (1) is exactly controllable from $\mathcal{S}$ in time $T>0$. Let $\Sigma_{s}, s \in(0,1]$, be a convex foliation satisfying (F1)-(Fr), let $s \in(0,1]$, and let $\kappa_{0} \in$ $C^{\infty}\left(\overline{\Omega_{s}}\right)$ be nowhere vanishing. Then there is a neighborhood $\mathcal{U}_{s} \subset M_{s}$ of $\Sigma_{s}$ such that the condition

$$
\mathcal{T}_{1, \Omega_{s}, 1}=\mathcal{T}_{2, \Omega_{s}, \kappa_{0}}
$$

implies that there exists $\kappa \in C^{\infty}\left(\mathcal{U}_{s}\right)$ such that

$$
\kappa(x) \neq 0, x \in \mathcal{U}_{s}
$$

and

$$
\left.\mathcal{A}_{1}\right|_{\mathcal{U}_{s}}=\left.\kappa^{-1} \mathcal{A}_{2} \kappa\right|_{\mathcal{U}_{s}} .
$$


Proof. For $j=1,2$, we fix $K_{j, \Omega_{s}, \kappa}$ given by (9) for $B=\Omega_{s}$ and $\mathcal{T}_{\Omega_{s}, \kappa}=$ $\mathcal{T}_{j, \Omega_{s}, \kappa}$ and applying (37) we deduce that

$$
K_{1, \Omega_{s}, 1}^{T}=K_{2, \Omega_{s}, \kappa_{0}}^{T} .
$$

Let $\mathcal{K} \subset \Sigma_{s}$ be compact, and consider the sets defined in Proposition 1 with $M$ replaced by $M_{s}$, $\Gamma$ replaced by $\Sigma_{s}$. We fix $U(\mathcal{K})$ the neighborhood of $\mathcal{K}$ in $M_{s}$ satisfying the properties of Proposition 1 . For all $p=(s, y) \in U(\mathcal{K})$, we define $\mathcal{B}_{p}(\mathcal{K})=\mathcal{B}\left(\Omega_{s}, \widetilde{h}, T\right)$ (see the beginning of Section 2.2 for the definition of this set), where $\widetilde{h}$ is as in Lemma 9 with the choice $h=(s+\epsilon) 1_{\Gamma_{p}(\mathcal{K})}$, and $\epsilon$ is as in Proposition 1. For all $H \in C_{0}^{\infty}\left(\mathcal{B}_{p}(\mathcal{K})\right)$ and $j=1,2$, we denote by $w_{j, H}$ the solution of (11) with $A=A_{j}, q=q_{j}$. We will start by proving that, for all $p=(s, y) \in U(\mathcal{K})$ there exists $\kappa \in C^{\infty}\left(C_{p}(\mathcal{K})\right)$ such that the following identity holds

$$
w_{1, H}(T, x)=\kappa(x) w_{2, H}(T, x),
$$

for $H \in C_{0}^{\infty}\left(\mathcal{B}_{p}(\mathcal{K})\right)$ and $x \in C_{p}(\mathcal{K})$. For any $x \in C_{p}(\mathcal{K})$, we consider functions $h_{k, x}$ and sets $X_{k, x}$ satisfying the properties described in Proposition 1 with $\mathcal{X}=M_{s}\left(\Omega_{s}, \widetilde{h}\right)^{\text {int }}$. Analogously to the proof of Theorem 1, we use Lemma 2 together with Lemma 9, (13) and Proposition 2. with $X_{k}=X_{k, x}$, to define $f_{k, x} \in \mathcal{C}_{0}^{\infty}((0,+\infty) \times S, k \in \mathbb{N}$, such that

$$
\begin{aligned}
w_{1, H}(T, x) & =\lim _{k \rightarrow+\infty}\left\langle u_{1, f_{k, x}}(T, \cdot), w_{1, H}(T, \cdot)\right\rangle_{L^{2}(M)} \\
& =\lim _{k \rightarrow+\infty}\left\langle u_{2, f_{k, x}}(T, \cdot), w_{2, H}(T, \cdot)\right\rangle_{L^{2}(M)}=\kappa_{x} w_{2, H}(T, x) .
\end{aligned}
$$

We introduce the function $\kappa: C_{p}(\mathcal{K}) \rightarrow \mathbb{C}$ by $\kappa(x)=\kappa_{x}$, and we get (40). Moreover, applying Lemma 8, we deduce that $\kappa$ is smooth and nowhere vanishing in $C_{p}(\mathcal{K}) \cup\left(\overline{C_{p}(\mathcal{K})} \cap \Sigma_{s}\right)$.

We consider now a collection $\left\{\mathcal{K}_{i} ; i \in I\right\}$ of compact sets in $\Sigma_{s}$ and $p_{i}=\left(s_{i}, y_{i}\right)$ lying in a neighborhood of $\mathcal{K}_{i}$ as in Proposition 1 . We assume that $\left\{\mathcal{K}_{i} ; i \in I\right\}$ and $\left\{p_{i} ; i \in I\right\}$ are chosen in such a way that $\bigcup_{i \in I} C_{p_{i}}\left(\mathcal{K}_{i}\right)$ is a neighborhood of $\Sigma_{s}$. Repeating the above argumentation, for all $i \in I$, we find $\kappa_{i} \in C^{\infty}\left(C_{p_{i}}\left(\mathcal{K}_{i}\right) \cup\left(\overline{C_{p_{i}}\left(\mathcal{K}_{i}\right)} \cap \mathcal{R}\right)\right)$ such that, for all $x \in C_{p_{i}}\left(\mathcal{K}_{i}\right)$, we have

$$
w_{1, H}(T, x)=\kappa_{i}(x) w_{2, H}(T, x), \quad H \in C_{0}^{\infty}\left(\mathcal{B}_{p_{i}}\left(\mathcal{K}_{i}\right)\right) .
$$

In a similar way to the end of the proof of Theorem 1 , applying Lemma 3. we can define $\kappa \in C^{\infty}\left(\bigcup_{i \in I} C_{p_{i}}\left(\mathcal{K}_{i}\right)\right)$ such that, for all $i \in I$, $\kappa_{\mid C_{p_{i}}\left(\mathcal{K}_{i}\right)}=\overline{\kappa_{i}}$. Combining this with (41), we can define $\mathcal{U}_{s}$ a neighborhood of $\Sigma_{s}$, such that (38) is fulfilled. 
4.2. Gluing of the gauges. Let $\mathcal{S}, \mathcal{R} \subset \partial M$ satisfy the assumptions of Theorem 2, and let $\Sigma_{s}, s \in(0,1]$, be a convex foliation satisfying (F1)-(F7). From now on, we assume that (4) is fulfilled and our goal is to prove (6). For this purpose, we define the set

$$
\begin{gathered}
J=\left\{s \in(0,1] ; \quad \text { there exists } U_{s} \subset M \text { an open set of } M\right. \\
\text { containing } \bar{\Omega}_{s} \text { such that } \\
\left.\left(\left.A_{1}\right|_{U_{s}},\left.q_{1}\right|_{U_{s}}\right) \in \mathcal{G}_{U_{s}, \mathcal{R}}\left(A_{2}, q_{2}\right)\right\}
\end{gathered}
$$

According to Theorem 1 and condition (F6), we know that $J \neq \emptyset$ since for $s$ small enough we have that $\overline{\Omega_{s}} \subset \mathcal{U}$, where $\mathcal{U}$ is a neighborhood of $\mathcal{R}$ as in Theorem 1. Moreover, the continuity condition (F5) implies that $J$ is open. Therefore, since $(0,1]$ is a connected set, the proof of Theorem 2 will be completed if we show that $J$ is closed. This will be our main task from now on. We start with four intermediate results.

Let $U \subset M^{\text {int }}$ be open. We define

$$
\mathbb{K}(U):=\left\{\kappa \in C^{\infty}(\bar{U}) ;\left.\kappa\right|_{\bar{U} \cap \mathcal{R}}=1, \kappa(x) \neq 0, x \in \bar{U}\right\} .
$$

Lemma 11. Let $U \subset M^{\text {int }}$ be open and connected and suppose that $\bar{U} \cap \partial M \subset \mathcal{R}$ and that the interior of $\bar{U} \cap \mathcal{R}$ in $\partial M$ is nonempty. Assume that there exists a piecewise smooth function $\kappa_{0}: U \rightarrow \mathbb{C}$ with the following properties:

(i) $\kappa_{0}(x) \neq 0$ for all $x \in U$,

(ii) there is a neighbourhood $W \subset M$ of $\mathcal{R}$ such that $\kappa_{0}$ is smooth in $U \cap W$ and extends smoothly to $\bar{U} \cap \mathcal{R}$,

(iii) the smooth extension satisfies $\kappa_{0}=1$ in $\bar{U} \cap \mathcal{R}$.

Suppose that, for $\mathcal{A}_{j}=\Delta_{g}-A_{j}-q_{j}, j=1,2$, the conditions (4) and

$$
\left.\mathcal{A}_{1}\right|_{U}=\left.\kappa_{0}^{-1} \mathcal{A}_{2} \kappa_{0}\right|_{U}
$$

are fulfilled. Then $\kappa_{0}$ is smooth and has a smooth extension $\kappa$ to $\bar{U}$. The smooth extension satisfies $\kappa \in \mathbb{K}(U)$ and the condition

$$
\mathcal{T}_{1, U, 1}=\mathcal{T}_{2, U, \kappa}
$$

is fulfilled.

Proof. We will divide the proof in four steps.

Step 1. We will show that (44) holds with $U$ replaced by a small subset of $U$ lying close to $\mathcal{R}$. As the interior of $\bar{U} \cap \mathcal{R}$ in $\partial M$ is nonempty, we can choose a nonempty open set $\Gamma \subset \bar{U} \cap \mathcal{R}$ and $r>0$ such that $M(\Gamma, r) \subset U$. For $j=1,2, f \in C_{0}^{\infty}((0,+\infty) \times S)$ and $\phi \in$ $C_{0}^{\infty}((T-r, T) \times \Gamma)$ we consider $u_{j, f}, v_{j, \phi}$ solving respectively (1), (10) with $A=A_{j}$ and $q=q_{j}$. By the finite speed of propagation (e.g. [21, 
Lemma 3.9]), we know that, for all $t \in[0, T], \operatorname{supp}\left(v_{2, \phi}(t, \cdot)\right) \subset M(\Gamma, r)$. Then, 43) and the uniqueness of solutions of (10) imply

$$
v_{1, \phi}=\left(\overline{\kappa_{0}}\right)^{-1} v_{2, \phi} \text {. }
$$

By Lemma 1, the condition (4) implies that

$$
\left\langle v_{1, \phi}(T, \cdot), u_{1, f}(T, \cdot)\right\rangle_{L^{2}(M)}=\left\langle v_{2, \phi}(T, \cdot), u_{2, f}(T, \cdot)\right\rangle_{L^{2}(M)}
$$

and combining this with 45 we get

$$
\left\langle v_{1, \phi}(T, \cdot), u_{1, f}(T, \cdot)-\kappa_{0} u_{2, f}(T, \cdot)\right\rangle_{L^{2}(M)}=0 .
$$

Then, using the density of the functions $v_{1, \phi}(T, \cdot), \phi \in C_{0}^{\infty}((T-r, T) \times$ $\Gamma)$, in $L^{2}(M(\Gamma, r))$, given by Lemma 2 , we find for $x \in M(\Gamma, r)$ that

$$
u_{1, f}(T, x)=\kappa_{0} u_{2, f}(T, x) .
$$

Now allowing $T>0$ to be arbitrary, we deduce that

$$
\mathcal{T}_{1, B, 1}=\mathcal{T}_{2, B, \kappa_{0}},
$$

where $B=M(\Gamma, r)^{\text {int }}$.

Step 2. Supposing that $p \in U$ and $\epsilon>0$ satisfy $B(p, 2 \epsilon) \subset U$, we will show that

$$
\mathcal{T}_{1, B(p, \epsilon), 1}=\mathcal{T}_{2, B(p, \epsilon), \kappa_{0}}
$$

implies

$$
\mathcal{T}_{1, B(p, 2 \epsilon), 1}=\mathcal{T}_{2, B(p, 2 \epsilon), \kappa_{0}} .
$$

For $H \in C_{0}^{\infty}((0,+\infty) \times B(p, \epsilon))$, we consider $w_{j, H}$ solving (11) with $A=A_{j}$ and $q=q_{j}$. Applying Lemma 1 and $(48)$, for $H \in C_{0}^{\infty}((0, \infty) \times$ $B(p, \epsilon)), f \in C_{0}^{\infty}((0, \infty) \times \mathcal{S})$, we get

$$
\left\langle w_{1, H}(T, \cdot), u_{1, f}(T, \cdot)\right\rangle_{L^{2}(M)}=\left\langle w_{2, \overline{\kappa_{0}} H}(T, \cdot), u_{2, f}(T, \cdot)\right\rangle_{L^{2}(M)} .
$$

On the other hand, for $H \in C_{0}^{\infty}((T-\epsilon, T) \times B(p, \epsilon))$, the finite speed of propagation and (43) imply $w_{2, \overline{\kappa_{0}} H}(T, \cdot)=\overline{\kappa_{0}} w_{1, H}(T, \cdot)$. Then we have

$$
\left\langle w_{1, H}(T, \cdot), u_{1, f}(T, \cdot)-\kappa_{0} u_{2, f}(T, \cdot)\right\rangle_{L^{2}(M)}=0
$$

and using the density of the functions $w_{1, H}(T, \cdot), H \in C_{0}^{\infty}((T-\epsilon, T) \times$ $B(p, \epsilon))$, in $L^{2}(M(B(p, \epsilon), \epsilon))$, given by Lemma 2 , we find

$$
u_{1, f}(T, x)=\kappa_{0} u_{2, f}(T, x), \quad x \in B(p, 2 \epsilon) .
$$

Allowing $T>0$ to be arbitrary, we get (49).

Step 3. We will show that $\mathcal{T}_{1, U, 1}=\widetilde{\mathcal{T}}_{2, U, \kappa_{0}}$. Let $p \in U$ and $p^{\prime} \in$ $M(\Gamma, r)$ int and connect $p$ to $p^{\prime}$ with a path $\gamma:[0,1] \rightarrow U$. Then there is $\epsilon>0$ such that $B(\gamma(t), 2 \epsilon) \subset U$ for all $t \in[0,1]$ and $B(\gamma(0), \epsilon) \subset$ $M(\Gamma, r)^{\text {int }}$. Now we can iteratively prove that

$$
\mathcal{T}_{1, B(\gamma(t), \epsilon), 1}=\mathcal{T}_{2, B(\gamma(t), \epsilon), \kappa_{0}}, \quad t \in[0,1] .
$$


Since $p \in U$ can be chosen arbitrarily, we deduce that $\mathcal{T}_{1, U, 1}=\mathcal{T}_{2, U, \kappa_{0}}$.

Step 4. We will show that $\kappa_{0}$ is smooth and that it has a smooth, nowhere vanishing extension to $\bar{U}$. Let $x_{0} \in \bar{U} \cap M^{\text {int }}$. By Lemma 3 there is $f \in C_{0}^{\infty}((0, \infty) \times \mathcal{S})$ and a neighborhood $B \subset M^{\text {int }}$ of $x_{0}$ such that $u_{2, f}(T, x) \neq 0$ for $x \in B$. Therefore,

$$
\kappa_{0}(x)=\frac{u_{1, f}(T, x)}{u_{2, f}(T, x)}, \quad x \in B \cap U .
$$

As $u_{j, f}(T, \cdot) \in C^{\infty}(\bar{U})$ for both $j=1,2$, this implies that $\kappa_{0}$ is smooth in $B \cap U$ and has a smooth extension to $B \cap \bar{U}$. By varying $x_{0}$, we see that $\kappa_{0}$ has a smooth extension to $\bar{U} \cap M^{\text {int }}$. Recalling also the assumption (ii), we see that $\kappa_{0}$ has a smooth extension to whole $\bar{U}$. The extension is unique and we denote it by $\kappa$. The smoothness of $\kappa$ and (46) for $x \in U$ imply that

$$
u_{1, f}(T, x)=\kappa(x) u_{2, f}(T, x),
$$

for all $x \in \bar{U}$ and all $f \in C_{0}^{\infty}((0, \infty) \times \mathcal{S})$. To see that $\kappa$ is nowhere vanishing in $\bar{U}$, let $x \in \bar{U} \cap M^{\text {int }}$ and choose $f \in C_{0}^{\infty}((0, \infty) \times \mathcal{S})$ such that $u_{1, f}(T, x) \neq 0$ (using Lemma 3 again). Now (50) implies that $\kappa(x) \neq 0$.

A direct consequence of this last result is given by the following.

Corollary 1. Let $s \in J$ where $J$ is defined by (42). Then (4) implies that there exists $\kappa_{s} \in \mathbb{K}\left(\Omega_{s}\right)$ such that

$$
\left.\mathcal{A}_{1}\right|_{\Omega_{s}}=\left.\kappa_{s}^{-1} \mathcal{A}_{2} \kappa_{s}\right|_{\Omega_{s}}, \quad \mathcal{T}_{1, \Omega_{s}, 1}=\mathcal{T}_{2, \Omega_{s}, \kappa_{s}} .
$$

Let us also consider the following result which will be important for the gluing of the gauge class.

Lemma 12. Let $s_{1}, s_{2} \in J$ where $J$ is defined by (42), and suppose that $s_{1}<s_{2}$. Then (4) implies that there exist $\kappa_{\ell} \in \mathbb{K}\left(\Omega_{s_{\ell}}\right), \ell=1,2$, such that $\left.\kappa_{2}\right|_{\Omega_{s_{1}}}=\kappa_{1}$ and such that the condition

$$
\left.\mathcal{A}_{1}\right|_{\Omega_{s_{\ell}}}=\left.\kappa_{\ell}^{-1} \mathcal{A}_{2} \kappa_{\ell}\right|_{\Omega_{s_{\ell}}}, \quad \mathcal{T}_{1, \Omega_{s_{\ell}}, 1}=\mathcal{T}_{2, \Omega_{s_{\ell}}, \kappa_{\ell}}, \quad \ell=1,2
$$

is fulfilled.

Proof. By Corollary 1 there exist $\kappa_{\ell} \in \mathbb{K}\left(\Omega_{s_{\ell}}\right), \ell=1,2$, such that (51) is fulfilled and the proof will be completed if we can show that $\left.\kappa_{2}\right|_{\Omega_{s_{1}}}=\kappa_{1}$. For this purpose, we remark that 51 implies

$$
\mathcal{T}_{2, \Omega_{s_{1}}, \kappa_{1}} f(x)=\mathcal{T}_{2, \Omega_{s_{2}}, \kappa_{2}} f(x), \quad f \in C_{0}^{\infty}((0, \infty) \times \mathcal{S}), x \in \Omega_{s_{1}} .
$$

We fix $x \in \Omega_{s_{1}}$. By Lemma 3 there is $f \in C_{0}^{\infty}((0, \infty) \times \mathcal{S})$ and a neighborhood $B \subset M^{\text {int }}$ of $x$ such that $u_{2, f}(T, x) \neq 0$ in $B$, where $u_{2}$ is the solution of (1) for $A=A_{2}$ and $q=q_{2}$. Thus $\kappa_{1}=\kappa_{2}$ in $B \cap \Omega_{s_{1}}$ 
and allowing $x \in \Omega_{s_{1}}$ to be arbitrary, we deduce that $\left.\kappa_{2}\right|_{\Omega_{s_{1}}}=\kappa_{1}$. This completes the proof of the lemma.

Lemma 13. Let $\left(s_{\ell}\right)_{\ell \in \mathbb{N}}$ be a strictly increasing sequence of $\mathbb{R}$ such that $s_{\ell} \in J, \ell \in \mathbb{N}$, and suppose that $\lim _{\ell \rightarrow \infty} s_{\ell}=s$. Here $J$ is defined by (42). Then (4) implies that there exists $\kappa \in \mathbb{K}\left(\Omega_{s}\right)$ such that

$$
\left.\mathcal{A}_{1}\right|_{\Omega_{s}}=\left.\kappa^{-1} \mathcal{A}_{2} \kappa\right|_{\Omega_{s}}, \quad \mathcal{T}_{1, \Omega_{s}, 1}=\mathcal{T}_{2, \Omega_{s}, \kappa} .
$$

Proof. An induction using Corollary 1 and Lemma 12 shows that, for all $\ell \in \mathbb{N}$, there exists $\kappa_{\ell} \in \mathbb{K}\left(\Omega_{s_{\ell}}\right)$, such that the following conditions are fufilled

$$
\begin{gathered}
\left.\mathcal{A}_{1}\right|_{\Omega_{s_{\ell}}}=\left.\kappa_{\ell}^{-1} \mathcal{A}_{2} \kappa_{\ell}\right|_{\Omega_{s_{\ell}}}, \quad \mathcal{T}_{1, \Omega_{s_{\ell}}, 1}=\mathcal{T}_{2, \Omega_{s_{\ell}}, \kappa_{\ell}}, \quad \ell \in \mathbb{N}, \\
\left.\kappa_{\ell+1}\right|_{\Omega_{s_{\ell}}}=\kappa_{\ell}, \quad \ell \in \mathbb{N} .
\end{gathered}
$$

According to (53), the functions $\kappa_{\ell}, \ell \in \mathbb{N}$, fit together and give a function $\kappa_{\infty}$ on $\Omega_{s}$ defined by

$$
\left.\kappa_{\infty}\right|_{\Omega_{s_{\ell}}}=\kappa_{\ell}, \quad \ell \in \mathbb{N}
$$

Moreover, by (52) we have

$$
\left.\mathcal{A}_{1}\right|_{\Omega_{s}}=\left.\kappa_{\infty}^{-1} \mathcal{A}_{2} \kappa_{\infty}\right|_{\Omega_{s}} .
$$

Here we recall that $\kappa_{\infty}$ is smooth in $\Omega_{s}$, up to $\overline{\Omega_{s}} \cap \mathcal{R}, \kappa_{\infty}$ is nowhere vanishing in $\Omega_{s}$ and satisfies $\kappa_{\infty}=1$ in $\overline{\Omega_{s}} \cap \mathcal{R}$. Now Lemma 11 implies that $\kappa_{\infty}$ has a smooth extension $\kappa \in \mathbb{K}\left(\Omega_{s}\right)$.

Armed with the above lemmas, we are now in position to complete the proof of the global result.

Proof of Theorem 2. It remains to show that $J$ is closed. Let $\left(s_{\ell}\right)_{\ell \in \mathbb{N}}$ be a strictly increasing sequence of $\mathbb{R}$ such that $s_{\ell} \in J, \ell \in \mathbb{N}$, and suppose that $\lim _{\ell \rightarrow \infty} s_{\ell}=s$. We will show that $s \in J$. By Lemma 13 , there exists $\kappa_{0} \in \mathbb{K}\left(\Omega_{s}\right)$ such that

$$
\left.\mathcal{A}_{1}\right|_{\Omega_{s}}=\left.\kappa_{0}^{-1} \mathcal{A}_{2} \kappa_{0}\right|_{\Omega_{s}}, \quad \mathcal{T}_{1, \Omega_{s}, 1}=\mathcal{T}_{2, \Omega_{s}, \kappa_{0}} .
$$

Combining this with Lemma 10, we deduce that there exist a neighborhood $\mathcal{U}_{s} \subset M_{s}$ of $\Sigma_{s}$ and $\kappa_{1} \in C^{\infty}\left(\mathcal{U}_{s}\right)$ such that

$$
\left.\mathcal{A}_{1}\right|_{\mathcal{U}_{s}}=\left.\kappa_{1}^{-1} \mathcal{A}_{2} \kappa_{1}\right|_{\mathcal{U}_{s}} .
$$

Combining this with (54), we obtain that for $\kappa$ defined by

$$
\kappa(x)= \begin{cases}\kappa_{0}(x), & x \in \Omega_{s}, \\ \kappa_{1}(x), & x \in \mathcal{U}_{s},\end{cases}
$$


and for $U_{s}=\Omega_{s} \cup \mathcal{U}_{s}$ we have

$$
\left.\mathcal{A}_{1}\right|_{U_{s}}=\left.\kappa^{-1} \mathcal{A}_{2} \kappa\right|_{U_{s}} .
$$

It is immediate that $\kappa$ is piecewise smooth, and Lemma 11 implies then that $\kappa$ is in $\mathbb{K}\left(U_{s}\right)$. Thus $J$ is closed and since $J$ is also open in $(0,1]$, we deduce that $J=(0,1]$. In particular $1 \in J$ which completes the proof.

\section{Complementary Results}

In this section we show that instead of assuming exact controllability from $\mathcal{S}$ and strict convexity of $\mathcal{R}$, we may assume that exact controllability holds from either $\mathcal{S}$ or $\mathcal{R}$ and that one of them is strictly convex. In the case that exact controllability holds from the set that is also strictly convex, we need the additional assumption that all the points in $M$ can be reached from the other set in time $T$. More precisely, supposing that exact controllability holds from strictly convex $\mathcal{R}$, we assume that

$$
T>\max _{x \in M} d(x, \mathcal{S}) .
$$

Then we can determine $A$ and $q$, up to the gauge equivalence, near the strictly convex set $\mathcal{R}$ or $\mathcal{S}$.

Observe first that the adjoint of $\Lambda_{\mathcal{S}, \mathcal{R}}^{T}$ is $R \Lambda_{\mathcal{R}, \mathcal{S}}^{T} R$ where $R$ is the time-reversal $R \phi(t)=\phi(T-t)$. Thus Theorem 1 implies that we can determine the geometry and the lower order terms near $\mathcal{S}$ if it is strictly convex and exact controllability holds from $\mathcal{R}$.

We will show next that the conclusion of Theorem 1 holds when $\mathcal{R}$ is strictly convex, the wave equation (1) is exactly controllable from $\mathcal{R}$, and (55) holds. The fourth case, that is, exact controllability holds from strictly convex $\mathcal{S}$ and (55) holds with $\mathcal{S}$ replaced by $\mathcal{R}$, follows then again by transposition. The global uniqueness result in Theorem 2 can also be changed in the analogous manner.

We used the exact controllability only once in the proof of Theorem 1. namely when we invoked Proposition 2. Proposition 3 below will substitute Proposition 2 in the case when the exact controllability holds from $\mathcal{R}$ instead of from $\mathcal{S}$.

Proposition 3. Let $\mathcal{X} \subset M$ be open, $x \in \mathcal{X}$ and let $X_{k} \subset M, k \in \mathbb{N}$, be a sequence of neighborhoods of $x$ satisfying $\lim _{k \rightarrow \infty} \operatorname{diam}\left(X_{k}\right)=0$. Let $\psi_{0} \in C_{0}^{\infty}(\mathcal{X})$ satisfy $\psi_{0}(x) \neq 0$. Let $T>0$ and suppose that a sequence $\left(f_{k l}\right)_{k, l=1}^{\infty}$ of functions in $L^{2}((0, T) \times \mathcal{S})$ satisfies

(0) for all $k$, there is $\delta_{k} \in L^{2}(M)$ such that the sequence $\left(u_{f_{k l}}(T, \cdot)\right)_{l=1}^{\infty}$ converges weakly to $\delta_{k}$ in $L^{2}(M)$, 
(i) there is $C>0$ such that $\left\|\delta_{k}\right\|_{L^{2}(M)} \leq C\left|X_{k}\right|^{-1 / 2}$ for all $k$,

(ii) $\operatorname{supp}\left(\delta_{k}\right) \subset \bar{X}_{k} \cup(M \backslash \mathcal{X})$ for all $k$,

(iii) $\left(\left\langle\delta_{k}, \psi_{0}\right\rangle_{L^{2}(M)}\right)_{k=1}^{\infty}$ converges.

Then there is $\kappa \in \mathbb{C}$ such that $\left.\lim _{k \rightarrow \infty l \rightarrow \infty} \lim _{f_{k l}}(T, \cdot), \psi\right\rangle_{L^{2}(M)}=\kappa \psi(x)$ for all $\psi \in C_{0}^{\infty}(\mathcal{X})$.

Furthermore, if (55) holds, then there is a sequence $\left(f_{k l}\right)_{k, l=1}^{\infty}$ that satisfies (i)-(iii) and for which $\kappa=1$.

Proof. Let $\psi \in C_{0}^{\infty}(\mathcal{X})$. Then $\operatorname{supp}\left(\delta_{k} \psi\right) \subset \bar{X}_{k}$ and

$$
\left\langle\delta_{k}, \psi\right\rangle_{L^{2}(M)}=\psi(x)\left\langle\delta_{k}, 1\right\rangle_{L^{2}\left(X_{k}\right)}+R_{k},
$$

where the remainder term $R_{k}$ converges to zero as $k \rightarrow \infty$. This can be seen as in the proof of Proposition 2 since $\left\|\delta_{k}\right\| \leq C\left|X_{k}\right|^{-1 / 2}$ for all $k$. We choose $\psi=\psi_{0}$ and see that $\lim _{k \rightarrow \infty}\left\langle\delta_{k}, 1\right\rangle_{L^{2}\left(X_{k}\right)}$ exists. We denote the limit by $\kappa$. Thus for any $\psi \in C_{0}^{\infty}(\mathcal{X})$ it holds that

$$
\lim _{k \rightarrow \infty l \rightarrow \infty}\left\langle K_{\mathcal{R}}^{T} f_{k l}, \psi\right\rangle_{L^{2}((0, T) \times \mathcal{R})}=\lim _{k \rightarrow \infty}\left\langle\delta_{k}, \psi\right\rangle_{L^{2}(M)}=\kappa \psi(x) .
$$

Let us now assume that $T>\max _{x \in M} d(x, \mathcal{S})$. Then Lemma 2 implies that for each $k$ there is a sequence $\left(f_{k l}\right)_{l=1}^{\infty}$ in $L^{2}((0, T) \times \mathcal{S})$ such that $\left(u_{f_{k l}}(T, \cdot)\right)_{l=1}^{\infty}$ converges to $1_{X_{k}} /\left|X_{k}\right|$ in $L^{2}(M)$. Then the conditions (0), (ii) and (iii) hold.

Let us now outline how the proof of Theorem 1 needs to be changed when Proposition 2 is replaced by Proposition 3. Let $x \in C_{p}(\mathcal{K})$ let $X_{k, x}$ and $\mathcal{X}$ be as in the proof of Theorem 1. We use the shorthand notation $X_{k}=X_{k, x}$ and do not emphasize the dependence on $x$ in the notation below.

As in the proof of Proposition 3, let $\left(f_{k l}\right)_{k, l=1}^{\infty}$ in $L^{2}((0, T) \times \mathcal{S})$ be such that $\left(u_{1, f_{k l}}(T, \cdot)\right)_{l=1}^{\infty}$ converges to $1_{X_{k}} /\left|X_{k}\right|$ in $L^{2}(M)$. Note that

$$
\lim _{k \rightarrow+\infty} \lim _{l \rightarrow+\infty}\left\langle u_{1, f_{k l}}(T, \cdot), \psi\right\rangle_{L^{2}(M)}=\psi(x), \quad \psi \in C_{0}^{\infty}(\mathcal{X}) .
$$

We will show that the conditions (0)-(iii) of Proposition 3 are fulfilled with respect to $j=2$. Then Proposition 3 implies the following analogue of equation (31) in the proof of Theorem 1 .

$$
\left.\lim _{k \rightarrow \infty l \rightarrow \infty} \lim _{k, f_{k l}}(T, \cdot), \psi\right\rangle_{L^{2}(M)}=\kappa \psi(x), \quad \psi \in C_{0}^{\infty}(\mathcal{X}) .
$$

The rest of the proof of Theorem 1 is unchanged.

Analogously to (30), we have

$$
\left\langle u_{1, f_{k l}}(T, \cdot), v_{1, \phi}(T, \cdot)\right\rangle_{L^{2}(M)}=\left\langle u_{2, f_{k l}}(T, \cdot), v_{2, \phi}(T, \cdot)\right\rangle_{L^{2}(M)},
$$


for $\phi \in L^{2}((0, T) \times \mathcal{R})$. Therefore

$$
\lim _{l \rightarrow \infty}\left\langle u_{2, f_{k l}}(T, \cdot), v_{2, \phi}(T, \cdot)\right\rangle_{L^{2}(M)}=\left\langle 1_{X_{k}} /\left|X_{k}\right|, v_{1, \phi}(T, \cdot)\right\rangle_{L^{2}(M)} \cdot
$$

Recall that Hilbert spaces are sequentially weakly complete, see e.g. [39, Th. V.7]. Assuming exact controllability from $\mathcal{R}$, we have

$$
\left\{v_{2, \phi}(T, \cdot) ; \phi \in L^{2}((0, T) \times \mathcal{R})\right\}=L^{2}(M),
$$

and hence (57) implies that $\left(u_{2, f_{k l}}(T, \cdot)\right)_{l \in \mathbb{N}}$ converges weakly in $L^{2}(M)$. This establishes (0), and we denote the weak limit by $\delta_{k}$.

It follows from (57), using the Cauchy-Schwarz inequality, that

$$
\begin{aligned}
\left\langle\left|X_{k}\right|^{1 / 2} \delta_{k}, v_{2, \phi}(T, \cdot)\right\rangle_{L^{2}(M)} & =\left\langle 1_{X_{k}} /\left|X_{k}\right|^{1 / 2}, v_{1, \phi}(T, \cdot)\right\rangle_{L^{2}(M)} \\
& \leq\left\|v_{1, \phi}(T, \cdot)\right\|_{L^{2}(M)} .
\end{aligned}
$$

Thus (58) together with the uniform boundedness theorem implies that $\left(\left|X_{k}\right|^{1 / 2} \delta_{k}\right)_{k \in \mathbb{N}}$ is bounded in $L^{2}(M)$. Therefore (i) holds. Using again (57), we have

$$
\left\langle\delta_{k}, v_{2, \phi}(T, \cdot)\right\rangle_{L^{2}(M)}=\left\langle 1_{X_{k}} /\left|X_{k}\right|, v_{1, \phi}(T, \cdot)\right\rangle_{L^{2}(M)}=0
$$

for all $\phi \in C_{0}^{\infty}\left(\mathcal{B}\left(\mathcal{R}, h_{k, x} ; T\right)\right)$, since $v_{1, \phi}(T, \cdot)$ is supported in the set $M\left(\mathcal{R}, h_{k, x}\right)$ that is disjoint with $X_{k}$, see Proposition 1. Thus the density result of Lemma 2 implies (ii). Using once more (57), and recalling that $\mathcal{X}=M\left(B_{\partial M}(y, \epsilon), s+\epsilon\right)^{\text {int }}$, we have for $\phi \in C_{0}^{\infty}\left(\overline{\mathcal{B}}\left(B_{\partial M}(y, \epsilon), s+\epsilon ; T\right)\right)$,

$$
\lim _{k \rightarrow \infty} \lim _{l \rightarrow \infty}\left\langle u_{2, f_{k l}}(T, \cdot), v_{2, \phi}(T, \cdot)\right\rangle_{L^{2}(M)}=v_{1, \phi}(T, x) .
$$

Thus, condition (iii) of Proposition 3 is also fulfilled when $\phi$ is chosen so that $v_{2, \phi}(T, x) \neq 0$.

Acknowledgements. Y. Kian was supported by the French National Research Agency ANR (project MultiOnde) grant ANR-17-CE40-0029, M. Lassas by Academy of Finland, projects 263235, 273979 and 284715, Y. Kurylev by the Engineering and Physical Sciences Research Council (EPSRC), UK, grant EP/L01937X/1, and L. Oksanen by the EPSRC grant EP/L026473/1.

\section{REFERENCES}

[1] R. Alexander, and S. Alexander. Geodesics in Riemannian manifolds-withboundary. Indiana University Mathematics Journal, 30(4):481-488, 1981.

[2] M. Anderson, A. Katsuda, Y. Kurylev, M. Lassas, and M. Taylor. Boundary regularity for the Ricci equation, geometric convergence, and Gel'fand's inverse boundary problem. Invent. Math., 158(2):261-321, 2004.

[3] C. Bardos, G. Lebeau, and J. Rauch. Sharp sufficient conditions for the observation, control, and stabilization of waves from the boundary. SIAM J. Control Optim., 30(5):1024-1065, 1992. 
[4] M. I. Belishev. An approach to multidimensional inverse problems for the wave equation. Dokl. Akad. Nauk SSSR, 297(3):524-527, 1987.

[5] M. I. Belishev and Y. V. Kurylev. To the reconstruction of a Riemannian manifold via its spectral data (BC-method). Comm. Partial Differential Equations, 17(5-6):767-804, 1992.

[6] A. S. Blagoveščenskiı̌. The local method of solution of the nonstationary inverse problem for an inhomogeneous string. Trudy Mat. Inst. Steklov., 115:28-38, 1971.

[7] R. Bosi, Y. V. Kurylev and M. Lassas. Reconstruction and stability in Gel'fand's inverse interior spectral problem. Preprint arXiv:1702.07937, 2017.

[8] H. Brezis. Functional analysis, Sobolev spaces and partial differential equations. Springer Verlag, New York, 2010.

[9] N. Burq and P. Gérard. Condition nécessaire et suffisante pour la contrôlabilité exacte des ondes. C. R. Acad. Sci. Paris Sér. I Math., 325(7):749-752, 1997.

[10] P. Caro, P. Ola, and M. Salo. Inverse boundary value problem for Maxwell equations with local data. Comm. Partial Differential Equations, 34(1012):1425-1464, 2009.

[11] F. J. Chung. A partial data result for the magnetic Schrödinger inverse problem. Anal. PDE, 7(1):117-157, 2014.

[12] T. Daudé, N. Kamran, and F. Nicoleau. Non-uniqueness results for the anisotropic Calderon problem with data measured on disjoint sets. Preprint arXiv:1510.06559, 2015.

[13] D. Dos Santos Ferreira, C. E. Kenig, J. Sjöstrand, and G. Uhlmann. Determining a magnetic Schrödinger operator from partial Cauchy data. Comm. Math. Phys., 271(2):467-488, 2007.

[14] H. W. Engl, M. Hanke, and A. Neubauer. Regularization of inverse problems, volume 375 of Mathematics and its Applications. Kluwer Academic Publishers Group, Dordrecht, 1996.

[15] A. Greenleaf and G. Uhlmann. Local uniqueness for the Dirichlet-to-Neumann map via the two-plane transform. Duke Math. J., 108(3):599-617, 2001.

[16] C. Guillarmou and L. Tzou. Calderón inverse problem with partial data on Riemann surfaces. Duke Math. J., 158(1):83-120, 2011.

[17] O. Imanuvilov, G. Uhlmann, and M. Yamamoto. Partial Cauchy data for general second order elliptic operators in two dimensions. Publ. Res. Inst. Math. Sci., 48(4):971-1055, 2012.

[18] O. Y. Imanuvilov, G. Uhlmann, and M. Yamamoto. The Calderón problem with partial data in two dimensions. J. Amer. Math. Soc., 23(3):655-691, 2010.

[19] O. Y. Imanuvilov, G. Uhlmann, and M. Yamamoto. Inverse boundary value problem by measuring Dirichlet data and Neumann data on disjoint sets. Inverse Problems, 27(8):085007, 26, 2011.

[20] A. Katchalov and Y. Kurylev. Multidimensional inverse problem with incomplete boundary spectral data. Comm. Partial Differential Equations, 23(12):55-95, 1998.

[21] A. Katchalov, Y. Kurylev, and M. Lassas. Inverse boundary spectral problems, volume 123 of Monographs and Surveys in Pure and Applied Mathematics. Chapman \& Hall/CRC, Boca Raton, FL, 2001. 
[22] A. Katchalov, Y. Kurylev, and M. Lassas. Equivalence of time-domain inverse problems and boundary spectral problem Inverse problems, 20(2):419436, 2004.

[23] C. Kenig and M. Salo. The Calderón problem with partial data on manifolds and applications. Anal. PDE, 6(8):2003-2048, 2013.

[24] Y. Kian, L. Oksanen, E. Soccorsi, and M. Yamamoto. Global uniqueness in an inverse problem for time fractional diffusion equations J. Diff. Equat., 264:1146-1170, 2018.

[25] Y. Kurylev. An inverse boundary problem for the Schrödinger operator with magnetic field. J. Math. Phys., 36(6):2761-2776, 1995.

[26] Y. Kurylev and M. Lassas. Gelf'and inverse problem for a quadratic operator pencil. J. Funct. Anal., 176(2):247-263, 2000.

[27] Y. Kurylev, L. Oksanen, and G. P. Paternain. Inverse problems for the connection Laplacian. J. Differential Geom. (to appear). Preprint arXiv:1509.02645.

[28] Y. V. Kurylev and M. Lassas. The multidimensional Gel'fand inverse problem for non-self-adjoint operators. Inverse Problems, 13(6):1495-1501, 1997.

[29] I. Lasiecka, J.-L. Lions, and R. Triggiani. Nonhomogeneous boundary value problems for second order hyperbolic operators. J. Math. Pures Appl. (9), 65(2):149-192, 1986.

[30] M. Lassas and L. Oksanen. Inverse problem for the Riemannian wave equation with Dirichlet data and Neumann data on disjoint sets. Duke Math. J., 163(6):1071-1103, 2014.

[31] M. Lassas and L. Oksanen. Local reconstruction of a Riemannian manifold from a restriction of the hyperbolic Dirichlet-to-Neumann operator. In Inverse problems and applications, volume 615 of Contemp. Math., pages 223-231. Amer. Math. Soc., Providence, RI, 2014.

[32] B. O'Neill. Semi-Riemannian geometry, volume 103 of Pure and Applied Mathematics. Academic Press Inc., New York, 1983.

[33] Rakesh. Characterization of transmission data for Webster's horn equation. Inverse Problems, 16(2):L9-L24, 2000.

[34] V. A. Sharafutdinov. Integral geometry of tensor fields. Inverse and Ill-posed Problems Series. VSP, Utrecht, 1994.

[35] P. Stefanov and G. Uhlmann. Recovery of a source term or a speed with one measurement and applications. Trans. Amer. Math. Soc., 365(11):5737-5758, 2013.

[36] P. Stefanov, G. Uhlmann, and A. Vasy. Boundary rigidity with partial data. J. Amer. Math. Soc., 29: 299-332, 2016.

[37] P. Stefanov, G. Uhlmann, and A. Vasy. On the stable recovery of a metric from the hyperbolic dn map with incomplete data. Inverse Problems and Imaging, 10(4): 1141-1147, 2016.

[38] D. Tataru. Unique continuation for solutions to PDE's; between Hörmander's theorem and Holmgren's theorem. Comm. Partial Differential Equations, 20(56):855-884, 1995.

[39] K. Yosida. Functional analysis, volume 123 of Grundlehren der Mathematischen Wissenschaften. Springer-Verlag, Berlin-New York, 1980. 
Aix Marseille Univ, Université de Toulon, CNRS, CPT, Marseille, FRANCE

E-mail address: yavar.kian@univ-amu.fr

Department of Mathematics, University College London, Gower Street, London UK, WC1E 6BT

E-mail address: y.kurylev@ucl.ac.uk

University of Helsinki, Department of Mathematics and Statistics, P.O. Box 68, FI-00014, Finland

E-mail address: Matti.Lassas@helsinki.fi

Department of Mathematics, University College London, Gower Street, London UK, WC1E 6BT

E-mail address: 1.oksanen@ucl.ac.uk 